International Journal of Neural Systems, Vol. 29, No. 7 (2019) 1850058 (22 pages)

(C) The Author(s)

DOI: $10.1142 / \mathrm{S} 0129065718500582$

\title{
A Machine Learning Approach to Reveal the NeuroPhenotypes of Autisms
}

\author{
Juan M. Górriz*, Javier Ramírez, F. Segovia and Francisco J. Martínez \\ Department of Signal Theory, Networking and Communications \\ University of Granada, Granada 18071, Spain \\ *gorriz@ugr.es; jg825@cam.ac.uk \\ Meng-Chuan Lai \\ Centre for Addiction and Mental Health \\ and The Hospital for Sick Children, Toronto, Canada \\ Department of Psychiatry \\ University of Toronto, Toronto, ON M6J 1H4, Canada \\ Michael V. Lombardo \\ Department of Psychology \\ University of Cyprus, 2109 Aglantzia, Nicosia, Cyprus \\ Simon Baron-Cohen, MRC AIMS Consortium ${ }^{\dagger}$ and John Suckling \\ Department of Psychiatry, University of Cambridge \\ Cambridge, CB2 0SZ, UK
}

Accepted 8 December 2018

Published Online 18 February 2019

\begin{abstract}
Although much research has been undertaken, the spatial patterns, developmental course, and sexual dimorphism of brain structure associated with autism remains enigmatic. One of the difficulties in investigating differences between the sexes in autism is the small sample sizes of available imaging datasets with mixed sex. Thus, the majority of the investigations have involved male samples, with females somewhat overlooked. This paper deploys machine learning on partial least squares feature extraction to reveal differences in regional brain structure between individuals with autism and typically developing participants. A four-class classification problem (sex and condition) is specified, with theoretical restrictions based on the evaluation of a novel upper bound in the resubstitution estimate. These conditions were imposed on the classifier complexity and feature space dimension to assure generalizable results from the training set to test samples. Accuracies above $80 \%$ on gray and white matter tissues estimated from voxel-based morphometry (VBM) features are obtained in a sample of equal-sized high-functioning male and female adults with and without autism $(N=120, n=30 /$ group $)$. The proposed learning machine revealed how autism is modulated by biological sex using a low-dimensional feature space extracted from VBM. In addition, a spatial overlap analysis on reference maps partially corroborated predictions of the "extreme male brain" theory of autism, in sexual dimorphic areas.
\end{abstract}

Keywords: MRI; multiclass classification; upper bounds; autism; spatial overlap analysis.

\footnotetext{
* Corresponding author.

†See Appendix B.

This is an Open Access article published by World Scientific Publishing Company. It is distributed under the terms of the Creative Commons Attribution 4.0 (CC-BY) License. Further distribution of this work is permitted, provided the original work is properly cited.
} 


\section{Introduction}

The discovery of a characteristic pattern of structural brain differences associated with autism spectrum conditions (ASC) would be a major advance in our understanding of this complex and highly variable developmental disorder. Not only would it be a substrate upon which to build a systems narrative of autism, but from this starting point, it might be possible to run development "in reverse" to disentangle the roles of environmental and genetic risk factors in its etiology. Unfortunately, "inconsistent" is perhaps the most common adjective associated with the extant literature in this area.

Initial MRI observations focused on increased total brain, total tissue, and total lateral ventricle volumes in adults with autism,$\frac{1}{1}$ but subsequent meta-analyses, including MRI-derived measurements as well as head circumference and post-mortem brain weights, were only able to detect a significant casecontrol difference amongst $4-5$ year olds. $\stackrel{2}{ }$ More recent meta-analyses based on fully automated voxel measures of gray and white matter volumes have confirmed the absence of any large difference in adults in either total gray matter $(\mathrm{GM})^{33}$ or total white matter $(\mathrm{WM}), \frac{3}{3}$ or in cerebellar volume $\frac{4}{4}$ Thus, if there are differences in overall brain size, then they occur very early in life ${ }^{81}$ and are followed by a decrease in volume towards normative values during maturation in adolescence and early adulthood.

\subsection{Gray and white matter distributions in the autistic brain}

Our knowledge of localized changes in brain anatomy has historically come from GM and WM distributions estimated by voxel-based morphometry (VBM). Undergoing several iterations and improvements over time, VBM pipelines are automated, reliable $e^{\sqrt{6}}$ and statistically well behaved ${ }^{[7}$ The success of the VBM technique can be measured by the large number of wide-ranging longitudinally and crosssectionally designed studies, as well as consistent cross-study patterns of tissue differences characterizing disorders like schizophrenia,,$\frac{8}{}$ Alzheimer's disease ${ }^{78}$ and major depressive disorder $9|9| 10$

Whilst measures of global volume have generally pointed toward increases in the very early lives of those with autism, local comparisons of gray and white matter volume have variously implicated both regional increases and decreases. The greatest consistency that emerges from metaanalyses $11 \quad 13] 15 \quad 18 / 33 \mid 80$ is the large variance of both the primary literature and the outcomes of the derived meta-analyses, where the inclusion or exclusion of just a few primary sources can significantly alter the aggregated pattern of differences.

Explanations for the absence of a coherent narrative on the structural brain differences associated with autism have been suggested to arise from variety of origins. Whilst methodological differences vary between studies, $\frac{19}{19}$ similar variability in VBM pipelines has not hindered the observation of characteristic patterns in other disorders ${ }^{8[10}$ Autism is also highly heterogeneous. ${ }^{[2]}$ Indeed, under DSM-IV , four categories were contained within Autism Spectrum Disorder: Asperger's disorder, childhood disintegrative disorder and pervasive developmental disorder. It has been argued that no reliable clinical diagnosis has been made with these sub-types 21 and that no consistent biological substrate has been discovered that differentiates between them leading to a single diagnostic category of Autism Spectrum Disorder in DSM- $\sqrt{22}$ that subsumes the sub-types. Nevertheless, it is unlikely that there will be an observation that unites these disorders, and in fact greater diversity in phenotyping, perhaps down to the individual level, is argued to be more likely to identify an underlying neurobiology for autism ${ }^{23}$ Stratification does appears to yield sensitivity improvements; for example, children with regressive autism appear to have increases in brain size, whereas those with nonregressive autism are not associated with a significant size change ${ }^{24}$; and males and females with autism display different patterns of GM change. 27 What is almost certain is that should there be a true effect, it is spatially diffuse and generally of low effect size.

The main demographic features of ASC are its high prevalence, affecting $1 \%$ of the entire population, $29 \sqrt[31]{31}$ and the significant skewing of the gender ratio towards male individuals to give values of 2-3:1 male:female, $\stackrel{30}{32}$ and potentially contributing to the heterogeneous patterns of brain structure associated with the disorder. Previously, most studies of the biology of autism have focused predominantly on males ${ }^{33}$ with male:female ratios in research samples in the range 8-15:1. Studies of functional imaging modalities that undertook analyses in each 
sex independently have found widespread significant cross-sectional differences. ${ }^{34}$ Structural MRI studies of autism following similar stratification strategies have been successful in assessing the atypical brain areas that are shared and distinct across the sexes $\frac{[}{3}$ In a recent MRI study comprising highfunctioning male and female adults with autism,, 34 exploratory analyses based on a VBM univariate statistical framework uncovered substantial heterogeneity in the neurobiology of autism. Furthermore, stratification by sex can provide the empirical evidence to test predictions from the "extreme male brain" (EMB) theory of autism ${ }^{71}$ and other similar theories $^{744}$ that predict a relationship between autism and biological sexual differentiation.

\subsection{The machine learning approach}

A univariate analysis statistically processes single features independently. Features may be voxelwise comparisons, $\stackrel{65] 66}{r}$ regions of interest (ROIs), 67 or models of brain features such as multiple hypothesis testing on VBM estimates 44 and intrinsic curvature $\frac{46}{46}$ However, detecting a putative small magnitude, diffuse spatial pattern of differences in autism with a univariate approach is difficult, even in large datasets, $\frac{25,26}{2}$ due to its low sensitivity to this type of effect.

Recently, effort has been expended on developing multivariate predictive models of biological sex based on univariate differential patterns of brain structure, i.e. cortical thickness. ${ }^{38}$ In a sample of neurotypical males and females, these models were subsequently applied to males and females with autism. Multivariate approaches have also been applied to other imaging measures that are hypothesized to be altered in autism, including brain networks, ${ }^{39147}$ texture features, $\frac{40142 / 43}{2}$ and other voxel and regionwise decomposition techniques, ${ }^{48 / 4969179}$ In general, multivariate cross-sectional studies of autism with VBM estimates of tissue volumes perform somewhat better than univariate methods $\frac{25 / 28}{2}$

One of the major limitations of a multivariate computer-aided diagnosis (CAD) systems $45[82,84$ is the need for a sample size $(l)$ that is sufficiently large compared to the number of dimensions $d$ (predictors) 54 ; that is, $l \gg d$. Neuroimaging rarely satisfies this condition, thus the proposed learning machines must be designed and adapted to this hostile environment, otherwise control of the generalization ability of the learning process is arguably weak 5558 A solution to this problem may be achieved by the application of feature extraction/selection (FES) algorithms 54 with linear classifiers rather than defining a specific metric on the data, $\frac{85}{85}$ or by evaluating the role that each dimension plays in the development of the machine learning architecture. ${ }^{50}$ The aim is twofold: (i) to reduce the number of input dimensions, retaining the relevant information by measuring the importance of each dimension, and (ii) to control the complexity of the selected classifiers to avoid overfitting, 51152 reducing the false positive rate (type I error). In this way, linear regressors endowed with regularization (e.g. Lasso) have been very useful in removing variables of reduced relevance from the model architecture, enhancing the contribution of the remaining variables ${ }^{\sqrt[53]{3}}$ Nevertheless, regularization approaches work on the input space enforcing an aggressive sparsity that results in a reduction of the set of nonfiltered variables to a value comparable with the number of training instances, $\frac{53}{5}$ wich is particularly damaging in neuroimaging.

In this work, we propose a new multivariate methodology based on a one versus one group classification scheme working on a feature space. The proposed system is designed under theoretical conditions imposed on the classifier complexity and the dimensionality of the input pattern. Briefly, the method comprises a subject-specific and regionwise partial least squares (PLS) FES that is then used to identify differences from patterns of GM and WM derived from MRI in a factorial design with factors of sex (male and female) and condition (autism or control), specifically: (i) identification of potential sex-related risk and protective factors for autism; (ii) characterization of the four classes described in terms of sex and condition; and (iii) visualization of sexually dimorphic areas related to the probability of classification of autism in males and females.

This paper is organized as follows: Sec. 2 introduces the dataset and individual preprocessing steps implemented. In Sec. 3, the overall methodology is presented, including a distribution-free upper bound for the true error rate of a classifier, using the resubstitution error estimate. These theoretical considerations permit the definition of reliable statistical learning and validation schemes using linear Support 
Vector Machine (SVM) and FES algorithms. In the context of the previous section, we demonstrate how the use of these techniques provides a meaningful overlap analysis between group-difference maps for VBM comparisons. Later, in Sec. 4, the experimental design is outlined, along with a description of a complete set of experiments, including the results obtained in each experiment, a $t$-test for significance in the classification experiments, and finally the spatial overlap analysis of the derived PLS-based maps with the same $p$-value as the $t$-test maps. In Sec. 5 these results are discussed and critiqued, and finally in Sec. 6, conclusions are drawn.

\section{Materials: A Heterogenous, Multidimensional and Multiclass MRI Dataset}

Participants $(l=120)$ included 30 right-handed premenopausal females and 30 males with autism, along with 30 neurotypical females and 30 neurotypical males (see Table 1 and Ref. [34). All groups were matched for age (range: 18-49 years) and full-scale IQ. Participants with autism had a formal clinical diagnosis of International Classification of Diseases10 (World Health Organization, 1992) childhood autism or Asperger's syndrome, or Diagnostic and Statistical Manual of Mental Disorders-IV text revision (American Psychiatric Association, 2000) autistic disorder or Asperger's disorder assessed by a psychiatrist or clinical psychologist in the National Health Service, UK. For further details on the inclusion criteria and the rationale in the diagnostic algorithm cut-offs, please see Refs. 34 37. Exclusion criteria for all groups are also outlined in Ref. 34 and included a current diagnosis or history of psychotic disorders, substance-use disorders, severe head injury, genetic disorders associated with autism (e.g. fragile $\times$ syndrome, tuberous sclerosis), intellectual disability (i.e. IQ $<70$ ), hyperkinetic disorder, Tourette's disorder, or any other medical condition significantly affecting brain function (e.g. epilepsy). The neurotypical groups did not have autism either themselves or in their family history.

The dataset was collected by the UK Medical Research Council Autism Imaging Multicenter Study (MRC AIMS) and was acquired at the Autism Research Centre, University of Cambridge. Further recruitment details can be found elsewhere.343744

In addition, a larger multicenter male sample from the MRC AIMS project ${ }^{37}$ was also used in this paper for spatial overlap analysis. It consisted of 84 neurotypical adult males and 84 males with autism matched for age and full-scale IQ (see Ref. 37] for further details).

\section{Methodology}

The main goal of this section is twofold: (i) to provide a statistical framework (see Fig. 1) under the conditions detailed in Sec. 3.1 for a set of linear classifiers; and (ii) to relieve the curse of dimensionality encountered in machine learning algorithms processing high-resolution images of the brain. The aim of using such filter methods 68 is to obtain a lowdimensional set of features and then to reduce the empirical risk without increasing the capacity of the set of classifiers ( $\mathrm{VC}$ dimension, 57 or the number of separating dichotomies ${ }^{61}$ ) by means of a linear SVM.

\subsection{Upper bounds of error for machine learning in neuroimaging}

In a neuroimaging classification problem, a $d$-dimensional input pattern $\mathbf{x}$ is observed and the aim is to determine to which class or condition $y$ belongs to, considered as a random variable $k=$ $1, \ldots, K$. In general, given a training sample of $l$ pairs $\left(\mathbf{x}_{i}, y_{i}\right)$, the parametric set of functional dependencies $\{F(\mathbf{x}, \alpha)\}$, where $\alpha$ is a parameter defining the set of specific functions with cardinality equal to $N$,

Table 1. Demographics details of the dataset, group means with their standard deviation (sd).

\begin{tabular}{|c|c|c|c|c|c|c|}
\hline \multirow[b]{2}{*}{ Sex } & \multicolumn{3}{|c|}{ Autism } & \multicolumn{3}{|c|}{ Normal } \\
\hline & $M$ & $F$ & Total & $M$ & $F$ & Total \\
\hline$N$ & 30 & 30 & 60 & 30 & 30 & 60 \\
\hline Age (SD) & $27.2172(7.2847)$ & $27.8067(7.6322)$ & $27.5119(7.4029)$ & $28.1801(5.6219)$ & $27.4643(6.4572)$ & $27.8222(6.0133)$ \\
\hline
\end{tabular}




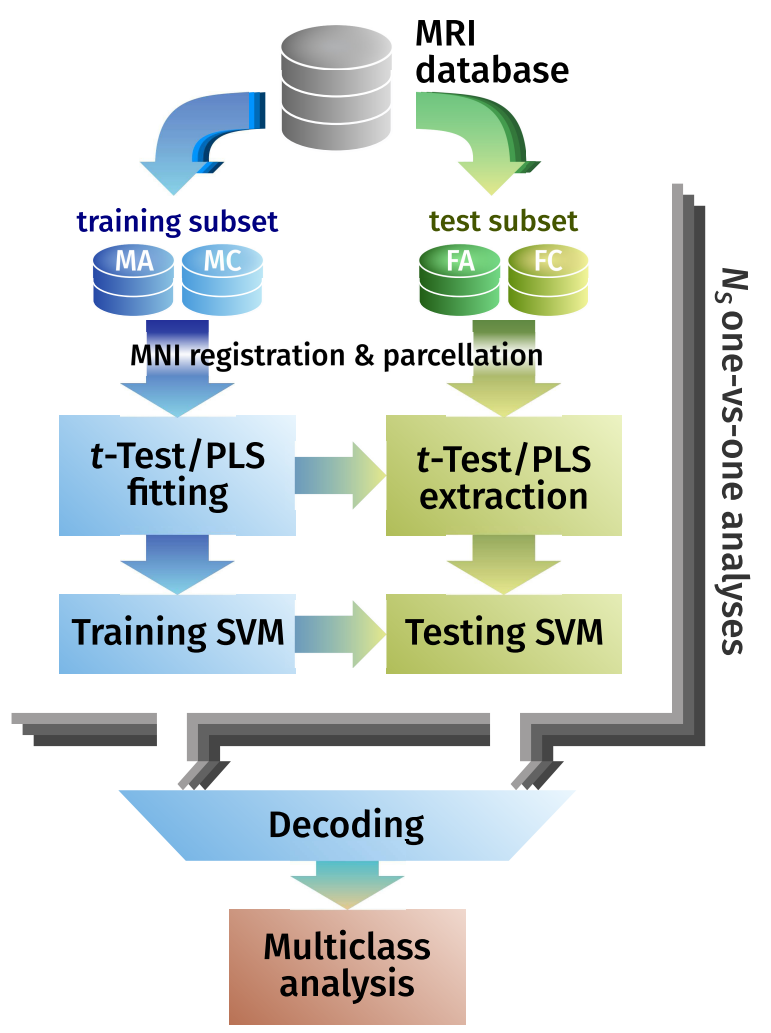

Fig. 1. Schematic representation of the proposed system. Given the $K=4$-class problem to be learned, $N_{s}=6$ different bipartitions are formed, and 6 binary problems (dichotomizers) over the partitions are trained and tested. As a result, a codeword of the same length is obtained for each class that is decoded by a Maximum A-Posteriori probability principle.

is required that minimizes the probability of misclassification. In other words, we minimize

$$
\begin{aligned}
P(F(\mathbf{x}, \alpha)) & \equiv P(F(\mathbf{x}, \alpha) \neq y) \\
& =\int_{y, X}(y-F(\mathbf{x}, \alpha))^{2} P(x, y) d x d y
\end{aligned}
$$

on the basis of the empirical data. In this case, the functional is known as the empirical risk and can be computed without knowing $P(\mathbf{x}, y)$ as

$$
P_{\mathrm{emp}}(F(\mathbf{x}, \alpha))=1 / l \sum_{i=1}^{l}\left(y_{i}-F\left(\mathbf{x}_{i}, \alpha\right)\right)^{2} .
$$

Let the minimum of Eq. (2) be attained for $F(\mathbf{x}$, $\left.\alpha_{\text {emp }}\right)$. The primary question is to establish when the decision rule $F\left(\mathbf{x}, \alpha_{\mathrm{emp}}\right)$ is close to $F\left(\mathbf{x}, \alpha_{o}\right)$, that which is obtained by minimizing Eq. (II). Following the derivation shown in the appendix, the actual risk obtainedby $\alpha_{\mathrm{emp}}$ is bounded by probability $1-\eta$ :

$$
P\left(\alpha_{\mathrm{emp}}\right) \leq P_{\mathrm{emp}}\left(\alpha_{\mathrm{emp}}\right)+\sqrt{\frac{1}{2 l} \ln \left(\frac{N}{\eta}\right)} .
$$

In addition, by the use of homogeneous linear threshold functions as decision rules $F(\mathbf{x}, \alpha \equiv \mathbf{w}): \mathbb{R}^{d} \rightarrow$ $\{-1,1\}$, where

$$
F(\mathbf{x}, \mathbf{w})= \begin{cases}1 & \text { if } \mathbf{x} \cdot \mathbf{w}>0 \\ -1 & \text { if } \mathbf{x} \cdot \mathbf{w}<0\end{cases}
$$

whenever the dimension (i.e. number of voxels/regions) of the input pattern $\mathbf{x}$ is far from the number of samples (i.e. scans), $d \ll l, P\left(\alpha_{\mathrm{emp}}\right)$ is seldom close to 0 and therefore the bound for the actual risk performs satisfactorily. In other words, for images of the brain where the number of scans is rather small compared to the number of voxels or regions, linear classifiers protect the system from overfitting, thus the variance of the actual risk is not large. Following the theory of homogeneous linear classifiers and applying the classical combinatorial geometry to develop the separating properties of decision surfaces, $\frac{55}{5}$ we can take a step forward and refine the bound in Eq. (3) as

$$
P\left(\alpha_{\mathrm{emp}}\right) \leq P_{\mathrm{emp}}\left(\alpha_{\mathrm{emp}}\right)+\sqrt{\frac{1}{2 l} \ln \left(\frac{N(l, d)}{\eta}\right)},
$$

where $N(l, d)$ is the number of linearly separable dichotomies of $l$ samples in a $d$-dimensional space (see further details in the appendix). Therefore, under the aforementioned conditions $(d \ll l)$, the empirical risk $P_{\text {emp }}\left(\alpha_{\mathrm{emp}}\right)$ is an indicator of the generalization ability of the statistical classifier, and the maximum deviation of the frequencies from the corresponding probability of the empirical solution $\left(\Delta P=P-P_{\mathrm{emp}}\right)$ can be derived with probability $1-\eta$ (see appendix).

\subsection{PLS-based CAD system}

Providing a significant set of features is important to achieving a small empirical risk given a classifier of fixed complexity (capacity). The binary FES and classification stages within the multiclass classification problem are performed in a class patternspecific manner, rather than using other strategies which combine different classes for subsequent fitting of binary statistical classifiers, i.e. the one versus rest-based model. In this way, given $K$ class patterns, a total number of $N_{s}=K(K-1) / 2$ binary 
classifiers may be fitted based on the same number of training subsets. This one versus one classification model (see Fig. 11) allows the assessment of which neuro-phenotypes defined in the $N_{s}$ training subsets are dominant in the corresponding $N_{s}$ test subsets. After that, the classification results can be merged to provide an overall classification result, defined as a decoding process in ternary error-correcting output code algorithms. ${ }^{[56}$ All of these analyses are achieved at a high confidence level given the upper bounds of the actual risk (see Sec. 3.1), the conditions regarding the sample size and the capacity of the selected statistical classifier.

\subsubsection{Structural magnetic resonance image acquisition and preprocessing}

All 120 participants were scanned using a contemporary $3 \mathrm{~T}$ MRI scanner (GE Medical Systems HDx) fitted with an 8-channel receive-only RT head-coil. Simulated T1-weighted inversion recovery images were segmented and normalized to the standard Montreal Neurological Institute (MNI) space using the SPM12 software (Wellcome Trust Centre for Neuroimaging, London, UK $)^{63}$ and the CAT12 toolbox ${ }^{64}$ Native space GM, WM and cerebro-spinal fluid (CSF) images were obtained using standard automated segmentation routines. The native space GM and WM images were registered to a studyspecific template using a high-dimensional nonlinear diffeomorphic registration algorithm (DARTEL) and then clustered into 116 standardized areas $\underline{62}$ A modulation step was included to retain voxelwise information about local tissue volume. The modulated GM and WM maps were smoothed with a $4 \mathrm{~mm}$ full-width at half-maximum Gaussian kernel. For the multicenter male sample, all preprocessing steps were developed in the same way as described earlier for the latter dataset. In the statistical inference part, the general linear model for VBM considered the sites (i.e. scanning machines) as covariates (categorical fixed-effect factors).

GM and WM maps $\mathbf{X}=\left\{\mathbf{x}_{i}\right\}$, for $i=1, \ldots, l$, were initially parcellated into $r=1, \ldots, 116$ standardized regions of a brain anatomical atlas. ${ }^{62}$ Thus, our multivariate analysis takes as its input mean gray and white matter volumes from within each atlas region, $\mathbf{x}_{i}(r)$, separately. This procedure is appropriate for our purpose of observing tissue-specific
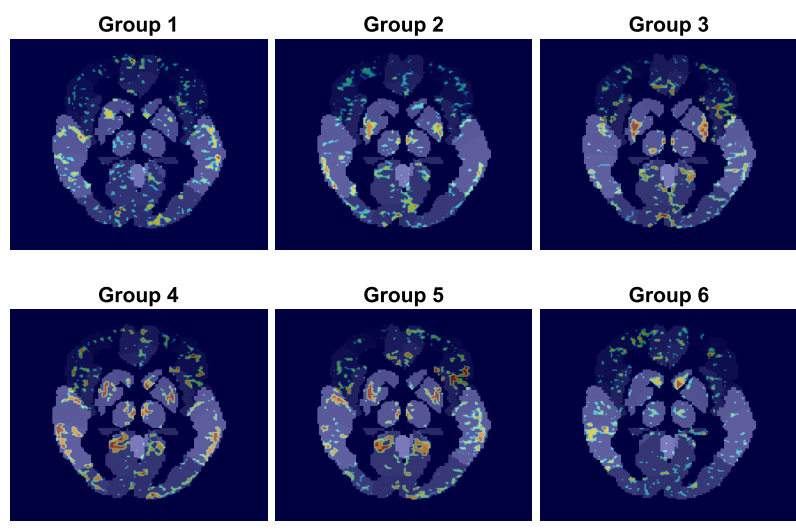

Fig. 2. (Color online) Significant GM differences (VBM) in the study groups $(p<0.05)$ plotted on the standardized atlas. Relative excesses in GM volume are displayed in orange/red, while deficits are displayed in blue (jet color map). From left to right, up to bottom: Male control (MC) versus Male Autism (MA); MC versus Female control (FC); MC versus Female Autism (FA); MA versus $\mathrm{FC}$; MA versus $\mathrm{FA}$; FC versus FA.
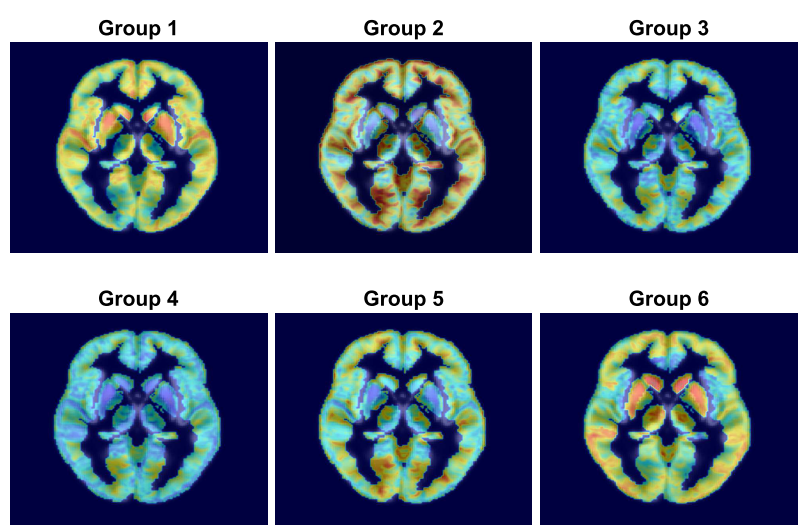

Fig. 3. (Color online) Brain map (PLS) illustrating regions where GM volume was associated with group membership (p loading). Orange/red regions indicate areas with a positive association between GM volume and condition (i.e. ASD > control); blue regions indicate brain systems of decreased GM volume in the condition group. The selected $z$-coordinate was 50 for the axial slice in the standard space of the MNI template.

local variations, although partial results may be rearranged by aggregation in a complete volume, as shown in the following sections and Figs. 2 and 3

\subsubsection{Two-sample t-test and PLS extraction}

A rank FES based on the standard two-sample $t$-test with pooled variance in combination with a PLSbased $\mathrm{FES}^{\sqrt{69}}$ was regionwise applied with the one 
versus one classification model (see Fig. 1). Given a training subset comprising two classes $j=1,2$ with balanced samples sizes $l_{1}=l_{2}=l / 2$, the $\mathbf{t}$ statistical vector is defined as

$$
\mathbf{t}=\frac{\overline{\mathbf{x}}^{1}-\overline{\mathbf{x}}^{2}}{\mathbf{s}_{p} \sqrt{\frac{4}{l}}},
$$

where $\mathbf{s}_{p}=\sqrt{\frac{s_{\mathbf{x}^{1}}+s_{\mathbf{x}^{2}}}{2}}$ is the pooled standard deviation and $s_{\mathbf{x}^{1}} s_{\mathbf{x}^{2}}$ are the unbiased estimators of the variances of the two classes (the assumption of the data in each group following a normal distribution was confirmed by a Kolmogorov-Smirnov test). The effect of this operation on the brain regions is to reduce the computational load prior to feature extraction, and to assure that regional differences in tissue volumes are considered when assessing overall patterns of tissue distribution.

Once the significant regions were identified by ranking the result of the $t$-test, we extracted the relevant patterns within those regions by a PLS regression between the $l \times d$ data matrix $\mathbf{X}$ and the $l \times 1$ vector labels $\mathbf{Y}$. Briefly, in the application of PLS to supervised classification, we maximized

$$
\omega_{o}=\max _{\omega}(\operatorname{cov}(\mathbf{X} \omega, \mathbf{Y}))^{2} ; \quad \text { s.t. }\|\omega\|=1,
$$

where the score vectors $\mathbf{s}=\mathbf{X} \omega$ were iteratively extracted and used to deflate the input matrix $\mathbf{X}$ by subtracting their rank-one approximations based on $\mathbf{s} . \frac{69}{}$ The deflation process was accomplished by the computation of the vector of loadings $\mathbf{p}$ as a coefficient of regressing $\mathbf{X}$ on $\mathbf{s}$ :

$$
\mathbf{p}=\frac{\mathbf{X}^{T} \mathbf{s}}{\mathbf{s}^{T} \mathbf{s}}=\mathbf{X}^{T} \hat{\mathbf{s}}
$$

The vector of weights $\omega_{f}$, where $f$ indexes the number of extracted features, is a local property in the images, that is, the dimensional components are not mixed in its computation, whilst the score coefficients $\mathbf{s}(i)=\sum_{j=1}^{d} X_{i j} \omega_{j}$ (and the matrix of scores), and the deflation term $\left.\mathbf{s p}^{T}\right|_{i j}=\frac{\sum_{k=1}^{d} s_{k} X_{i k} s_{i}}{\sum_{i=1}^{l} s_{i}^{2}}$, etc. are global. Therefore, the size of the input data $d$ is crucial to the assessment of the relationship between GM (WM) volume and group membership with heterogeneous variances, where some statistical properties of the involved processes, such as the stationarity or the ergodicity in the correlation, must be assumed for the evaluated ROIs. In the present study, the analysis was carried out on the set regions (see Figs. 2 and (3) selected by feature selection.

\subsubsection{Statistical parametric PLS maps}

The analysis of functional and structural studies usually entails the construction of spatially extended statistical processes where each voxel of the novel image or map is the result of a statistical test. After that hypothesis testing process, the main problem is to determine the significance of extrema by the use of several statistical field-based approximations, 14 i.e. a three-dimensional (3D) $t$-statistical field. PLS methods have been demonstrated in the past to be very useful in describing the relation between brain activity and experimental design or behavior measures ${ }^{41169}$ In this way, PLS analysis of brain activations is able to reveal additional regions of salience that are not identified by typical univariate voxelwise methods such as SPM. Assume that the label vector $\mathbf{Y}$ contains the two global conditions in $\mathbf{X}\left(\{1,-1\}\right.$ indexed as $\left.i \in C_{1}, C_{2}\right)$ and $\mathbf{x}_{i_{1}}>\mathbf{x}_{i_{2}}$, for $i_{1} \in C_{1}$ and $i_{2} \in C_{2}$, without loss of generalization. The scores $\mathbf{s}$ will be ideally located around zero with different signs, thus from Eq. (77) for every loading component, we have

$$
p(k)=\sum_{i_{1}} x_{i}(k) \hat{s}(i)-\sum_{i_{2}} x_{i}(k)|\hat{s}(i)|
$$

for $k=1, \ldots, d$, and a deviation from zero of this data-weighted score summation would suggest a region associated with a particular group membership (contrast). Remapped into image space, the contents of the singular vector $\mathbf{p}$ indicate which pixels are most sensitive to those predefined contrasts and define the so-called PLS map. Comparing the latter expression to Eq. (5), the PLS map can be seen as a multivariate two-sample test weighted by the scores of each subject with unknown distribution, except for the normalization term that depends on the pooled standard deviation. In fact, after some manipulations, it yields

$$
\mathbf{p}=\overline{\mathbf{x}}^{1}-\overline{\mathbf{x}}^{2},
$$

where $\overline{\mathbf{x}}^{j}(k) \equiv \sum_{C_{i}} x_{i}(k) P\left(x_{i}(k)\right)$ and $P\left(x_{i}(k)\right)=$ $|\hat{s}(i)|$ is the frequency of the observation $x_{i}(k)$ that is assumed to be proportional to its score in the computation of the group mean. 
The statistical significance of the PLS maps can be assessed is many ways, e.g. by the use of a permutation test ${ }^{41}$ In this work, we proposed the use of a parametric approach, that is very popular in neuroimaging, based on the Gaussian Random Field (GRF) theory. This theory models both the univariate probabilistic features of the resulting SPM and the nonstationary spatial covariance structure of that image $\frac{63}{6}$ This model can be applied as well to the proposed PLS map as the selected quantity that characterized the PLS computation which has shown to be very similar to the classical two-sample $t$-test mapping (except for the normalization factor). The methodology can be described as the following. First, given a $p$-value and the T SPM extracted from the one tailed two-sample $t$-test with contrast matrix $[1,-1]$ for $C_{1}$ and $C_{2}$ conditions, we determine the statistically significant positive salience threshold $t_{\text {critic }}$ using the inverse cumulative density function of the $t$-test distribution (the same applies for the negative one). The biased version of the PLSbased two-sample $t$-test in Eq. (9) makes up a novel P SPM that is linearly projected to a (bias corrected) distribution $\hat{\mathbf{P}}$ with the same parameters of the previous T SPM. ${ }^{a}$ Finally, the positive saliences on this novel map are determined by evaluating the regions, where $\hat{\mathbf{P}}>t_{\text {critic }}$ at the given $p$-value.

\subsubsection{A robust statistical classifier, SVM}

The use of SVMs was motivated by the minimization of the $\mathrm{VC}$ dimension and has been successfully shown to be a robust solution in classification learning, 58 which minimizes the separation margin between the binary-labeled training data, mapped into a (PLSbased) feature space $\mathfrak{F}$, by constructing a hyperplane w whose norm is minimum 58 :

$$
\|\mathbf{w}\|^{2}+C \sum_{i=1}^{l} \xi_{i}
$$

subject to

$$
y_{i}\left(\mathbf{w} \cdot \mathbf{x}_{i}\right) \geq 1-\xi_{i} ; \quad \xi_{i} \geq 0 ; \quad i=1, \ldots, l,
$$

\footnotetext{
${ }^{\mathrm{a}}$ Here, we assume that the $t$-distribution is approximately normal for a high number of degrees of freedom. Note that if a variable $X \sim \mathcal{N}\left(\mu_{1}, \sigma_{1}\right)$, then $Z=\left(X-\mu_{1}\right) /$ $\sigma_{1} \sim \mathcal{N}(0,1)$. Conversely, given $Z$, then $Y=\mu_{2} Z+\sigma_{2} \sim$ $\mathcal{N}\left(\mu_{2}, \sigma_{2}\right)$. Then, we can connect any pair of distributions by $Y=\frac{\sigma_{2}}{\sigma_{1}} X+\left(\mu_{2}-\mu_{1} \frac{\sigma_{2}}{\sigma_{1}}\right)$.
}

where $\xi_{i}$ are slack variables, $C$ is a constant that allows a trade-off between training error and model complexity ( $C$ is usually optimized by several searching methods at the training stage, i.e. Bayesian optimization in a wide range $[1 e-3,1 e 3])$, and the decision rule is defined as $F(\mathbf{x}, \mathbf{w})$ in Sec. 3.1 The solution is computed using $\mathbf{w}=\sum_{i=1}^{l} a_{i} y_{i} \mathbf{x}_{\mathbf{i}}$, where the multipliers $0 \leq a_{i} \leq C$ were derived from the dual Lagrangian problem in Eq. (10).

\section{Experiments and Results}

The spatial representation of the binary classifier output and the corresponding "loading" of the PLS image as the new reference base for the analyzed input patterns is discussed and shown in this section. In this sense, we take a step forward with respect to the majority of exploratory analyses developed so far in the literature ${ }^{34 / 44}$ and propose a specific cross-validation scheme with the purpose of discovering generalizable class features in the GM or WM images. By training the system using binary group differences (gender and/or condition) and under the assumption that they are distributed across statistically significant regions, we aim to generalize these features in the remaining study groups which should share the same magnitude of differences across groups. The complete analysis included a class pattern-specific two-sample test for regionwise FES, an overlap analysis 34 across group-difference PLS maps with a VBM comparison to evaluate the Autism theories, the assessment of the sample Autism label probability with the determination of confidence intervals using the Clopper-Pearson method and finally, permutation tests to check the statistical significance of the classification results obtained by the machine learning-based system.

\subsection{Experimental setup}

As discussed previously in Sec. 3.2 the images were parcellated according to Ref. 62 and FES algorithms were applied to the resulting images obtained from a standardized preprocessing and image registration pipeline ${ }^{64}$ For the sake of clarity, we define $N_{s}=6$ SVM classifiers acting on their corresponding group comparisons: Group 1 (G1): MC-MA (male controls versus male autistic individuals), G2: MC-FC (MC versus female controls), G3: MC-FA (MC versus 

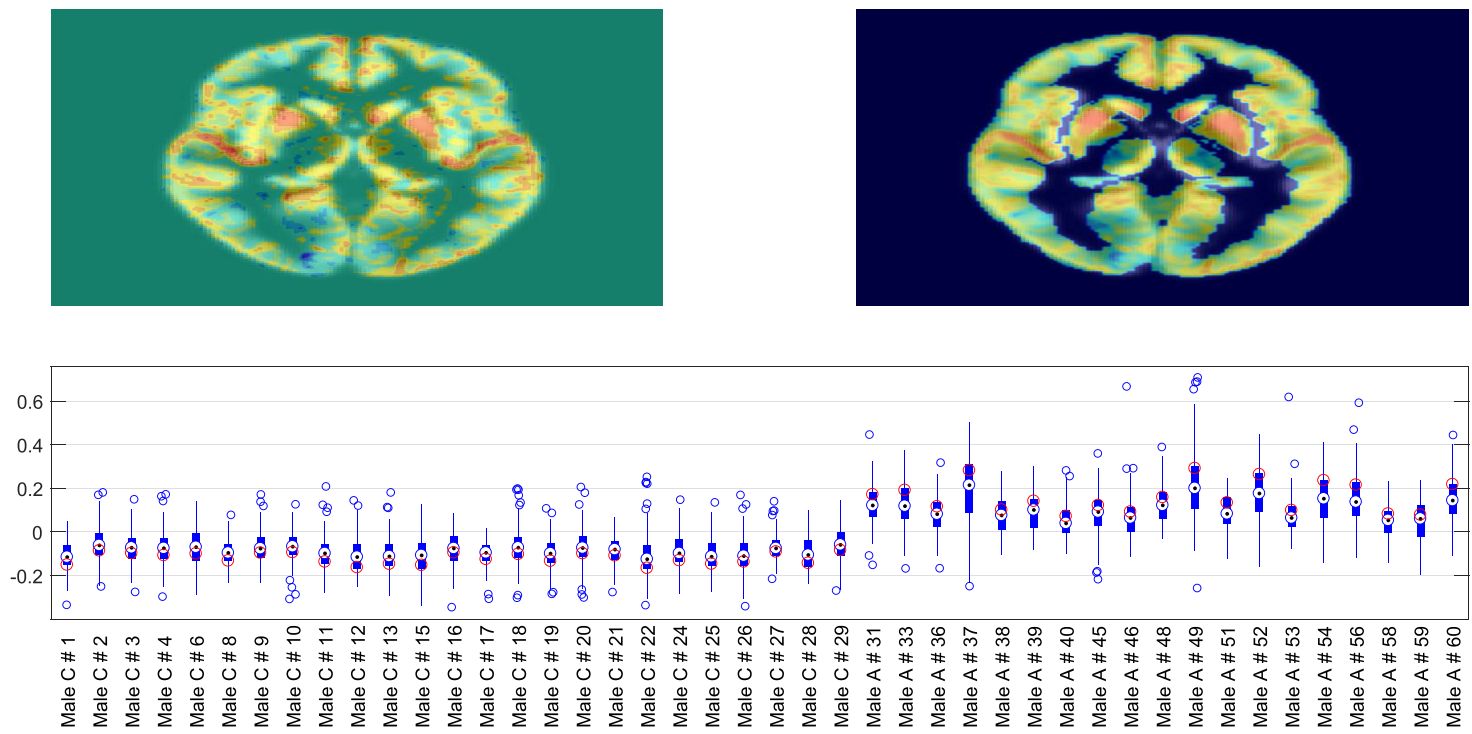

Fig. 4. Up: Detail of Slice \#50 for the p-loading in Group 1 under global (on the left) and regionwise (on the right) PLS FE. Note the noisy PLS loading on the left image as a result of processing the whole image simultaneously. Bottom: one-sample $t$-test on the scores $\mathbf{s}$, the rejection of the null hypothesis is obtained for 44 out of 60 subjects.

female autistic individuals), G4: MA-FC, G5: MAFA and $G 6$ : FC-FA.

\subsubsection{Preprocessing}

The application of the FES approach detailed in Sec. 3.2 results in several weight maps for the group comparisons as shown in Fig. 2

With the aim of increasing the generalization ability of the proposed classification systems, only the first PLS component was considered (a single dimension $d=1$ ), thus the associated upper bound of the actual risk, as shown in Eq. (1), provides a strong connection between the risk and the empirical risk (resubstitution error). It is worth mentioning what is actually shown in Figs. 3 and 4 is the first loading component $\mathbf{p}$ extracted from the brain regions, separately. Figure 3 highlights the relevant regions in terms of the PLS regression on the group differences. Group differences $G 1$ and $G 6$ illustrate the regions where the features associated with autism are different in males and females, respectively. In the remainder of the figures, features associated with gender are predominantly found in the PLS maps, e.g. the $G 2$ pattern that refers to the VBM comparison between $\mathrm{MC}$ and $\mathrm{FC}$ is modulated and found in group differences $G 3, G 4$, and $G 5$. The statistical significance of these PLS maps is discussed in Sec. 4.2 where the null distributions are modeled as a two-tailed Gaussian distribution or a $t$-distribution with large degrees of freedom $\nu$.

In Fig. 4 the PLS global approach is shown on the left and is contrasted against the regionwise comparison for $G 1$ (i.e. MC-MA). Observe that the regionwise comparison provides a noisy $\mathbf{p}$-loading that is used as a reference base in the feature space for the extracted input scores. A one-sample $t$-test between the score data $\mathbf{s}$ obtained from the regionwise approach and the global approach reveals that, at a $95 \%$ significance level, 44 out of the 60 subjects present a difference in means in regions including the insula, hippocampus, and cingulum; Fig. 4.

\subsubsection{Classification}

The overall classification accuracy of each SVM model was estimated using the re-substitution error for the training subset and a $l / 2$-holdout crossvalidation error for the test subset, testing for significance by repeating the validation procedure $n=500$ times, after randomly permuting the class labels. In this sense, each of $N_{s}=6$ training subsets, under the one versus one classification model, allows us to estimate the probability of the neurophenotype in terms of sex or condition and to evaluate it on the remaining $N_{s}$ test subsets. Therefore, the four classes 
are considered as a set of participants with different proportions or combinations of these two types of effect (sex and condition).

The selection of other cross-validation procedures, i.e. $K$-fold, with the aim of "pure" classification leads to poor classification and generalization of results mainly due to the variability of the input patterns, the potentially preponderant role of one effect, and small sample size. Thus, we should consider the former $K=4$ classes as tentative labels as they may contain spatially-dependent neurophenotypes with biases towards a particular effect that are nonuniformly distributed in the $K$ classes.

\subsection{Visualizing the patterns of brain regions representative of normative sex/condition differences}

The aim of this section is to analyze the differences of the specific patterns representing sex or condition. Following a similar analysis as in Ref. 34 we undertook a two-sample $t$-test based on VBM comparisons between groups. Only voxelwise height thresholds with no spatial extent operation were applied under the two logical contrasts, i.e. $\operatorname{group}_{1}>$ or $<$ group $_{2}$. Three sets of VBM comparisons were performed to test EMB theory predictions ${ }^{71}$; namely, MC-FC, MC-MA, and FC-FA comparisons. The aim of this procedure is to generate an overlap analysis across group-difference maps ${ }^{34}$ and compare them with the PLS maps. The PLS maps can be interpreted as two-tailed statistical tests, unlike the classical one-tailed $t$-test maps obtained under the SPM framework, $\frac{64}{6}$ and both are almost normally distributed with a high number of degrees of freedom (e.g. $\nu=57$ for the condition comparison) $]^{[70}$ In order to make them comparable for the computation of group-difference maps, given a set of $p$ values $\{0.0001, \ldots, 0.05\}$, we linearly transform the PLS maps, $P \sim \mathcal{N}\left(\mu_{p}, \sigma_{p}\right)$ to a new Gaussian distribution with mean and standard deviation of the corresponding comparison group $T$-maps, which are normally distributed $T \sim \mathcal{N}\left(\mu_{t}, \sigma_{t}\right)$. After this transformation, the PLS maps permit testing of the logical contrasts in a single map by $\operatorname{group}_{1} \neq$ group $_{2}$. As an example, in those regions where there is a statistically significant sexual dimorphism $\mathrm{MC} \neq \mathrm{FC}$, we evaluated the significant differences in the neurophenotype of male and female individuals with autism,

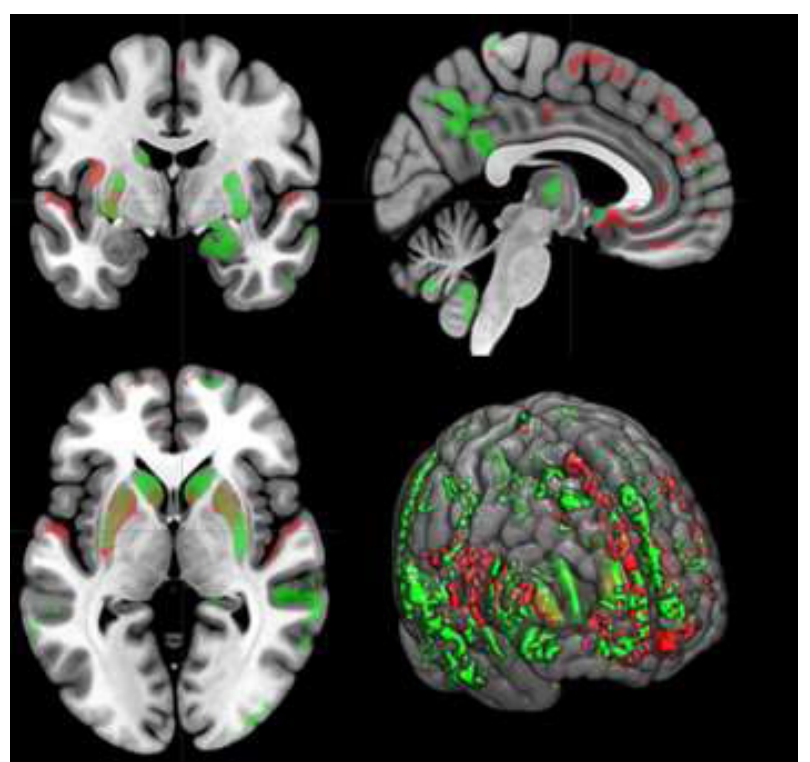

Fig. 5. (Color online) GM overlapping regions in sexually dimorphic areas of males (red) and female (green) individuals with autism at a voxel-level threshold of $p$-value $=0.05$.

that is, $\mathrm{MA} \neq \mathrm{MC}$ and $\mathrm{FA} \neq \mathrm{FC}$ (see Fig. 5 ). As shown in the following section, the overlap analysis derived from these figures partially support EMB theory predictions by considering both directions of effect at the same time $(>$ and $<)$.

\subsubsection{Spatial overlap analysis}

Following the discussion in Sec. 4.2 three VBM comparisons, MC-FC, MA-MC, and FA-FC, were evaluated on GM and WM volumes. We carried out the experiments on our dataset $(l=120)$ and the male sample $(l=168)$ described in Ref. 37, using our PLS-based FE approach and the set of one-tailed contrasts obtained from the univariate $2 \times 2$ factorial design analysis provided by the SPM software. ${ }^{63}$ For each comparison, a conjunction analysis ${ }^{3473}$ consisting of logical AND masking, were assessed and tested for significance by running Monte carlo simulations (5000 iterations). The distribution of overlaps at the same $p$-value threshold was sampled, i.e. from 0.0001 to 0.05 in steps of 0.0001 . This spatial overlap analysis, which considers synthetic GM (WM) map overlaps, allows us to assess the probability of whether the overlapping voxels of the comparisons occur by chance. In the baseline comparisons, we included an additional spatial overlap analysis using 

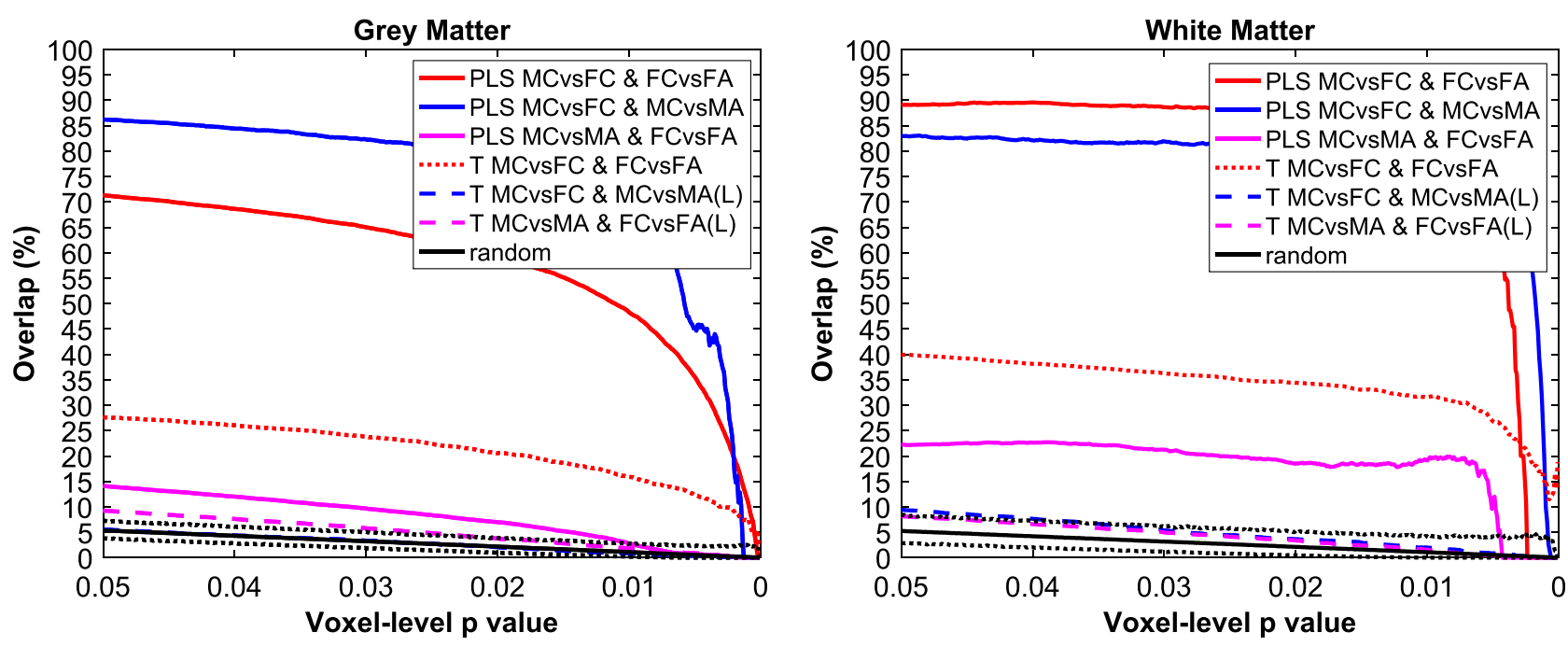

Fig. 6. Comparison of the testing brain-level predictions of the EMB theory of Autism. (L) indicates the use of the larger male database (i.e. using the DESPOT1 acquisitions) for the VBM comparison, described in Ref. 37

a multicenter male sample as detailed in Refs. 34 and 37. The purpose of this MA-MC comparison is to conduct the same analysis on a larger number of samples $(l=168)$ to provide higher power to detect the group differences, that were not found by the use of the current database ( $l=60$, males only).

The spatial overlap analysis between males and females with autism is small in both approaches ( $T$ and PLS maps) for GM and WM (e.g. up to $15 \%$ and $10 \%$ for GM and up to $23 \%$ and $10 \%$ for $\mathrm{WM}$, respectively) for $p \leq 0.05$ as shown in Fig. [6 in magenta color lines. This finding clearly identifies the differences between the neuroanatomy of autism across sexes that was previously demonstrated by the high precision of the four-class learning machine detailed in the previous section, and in Fig. 5 The rest of the curves show a significant difference between approaches, that is, the one-tailed $T$ maps versus the two-tailed-PLS maps. In the latter approach, we find significant evidence for overlap between structures sensitive to diagnosis and sexual dimorphism in both sexes (red and blue solid lines). This evidence is only found for the one-tailed $T$-based approach in females (T-MC-FC\&FC-FA, red dotted line in Fig. 国).

\subsection{Classification results}

The output score pattern of the set of binary classifiers is shown in Fig. 7 for the training and test sets. From this set of figures what is interesting to note is the ability for generalization of the proposed system, under some constrained conditions, over the tests $G 2$, G3, G4, and G5 (same rows on the figure). Another relevant feature found in the output score maps is the presence of vertical bars representing misclassification in training and test sets. This could be identification of the different neurophenotypes of specific individuals, e.g. participant 42 in the FC subset, as they are always located in the wrong hyperplane subspace at both training and test stages (see Table 2). From this table, it is clear that MA is the class with lower performance in classification accuracy in both stages. Surprisingly, the features associated with autism in the training of the SVM to classify $G 1$ (males) cannot be generalized in the test set (females) although these features should be present in one class, and absent in the other (a real binary classification problem). The almost random pattern found in the test set could indicate the different nature of the features of autism in males and females. This situation is repeated in $G 6$ where the females were used in the training stage and males made up the test set, confirming the latter hypothesis.

The quantitative analysis of the output score pattern is found in Fig. 8 where we represent the distribution of accuracies, including the notch analysis to display the variability of the median between samples, for all the brain regions and the accompanying overlay histograms. From this figure, we can observe how the learning systems generalize well with 

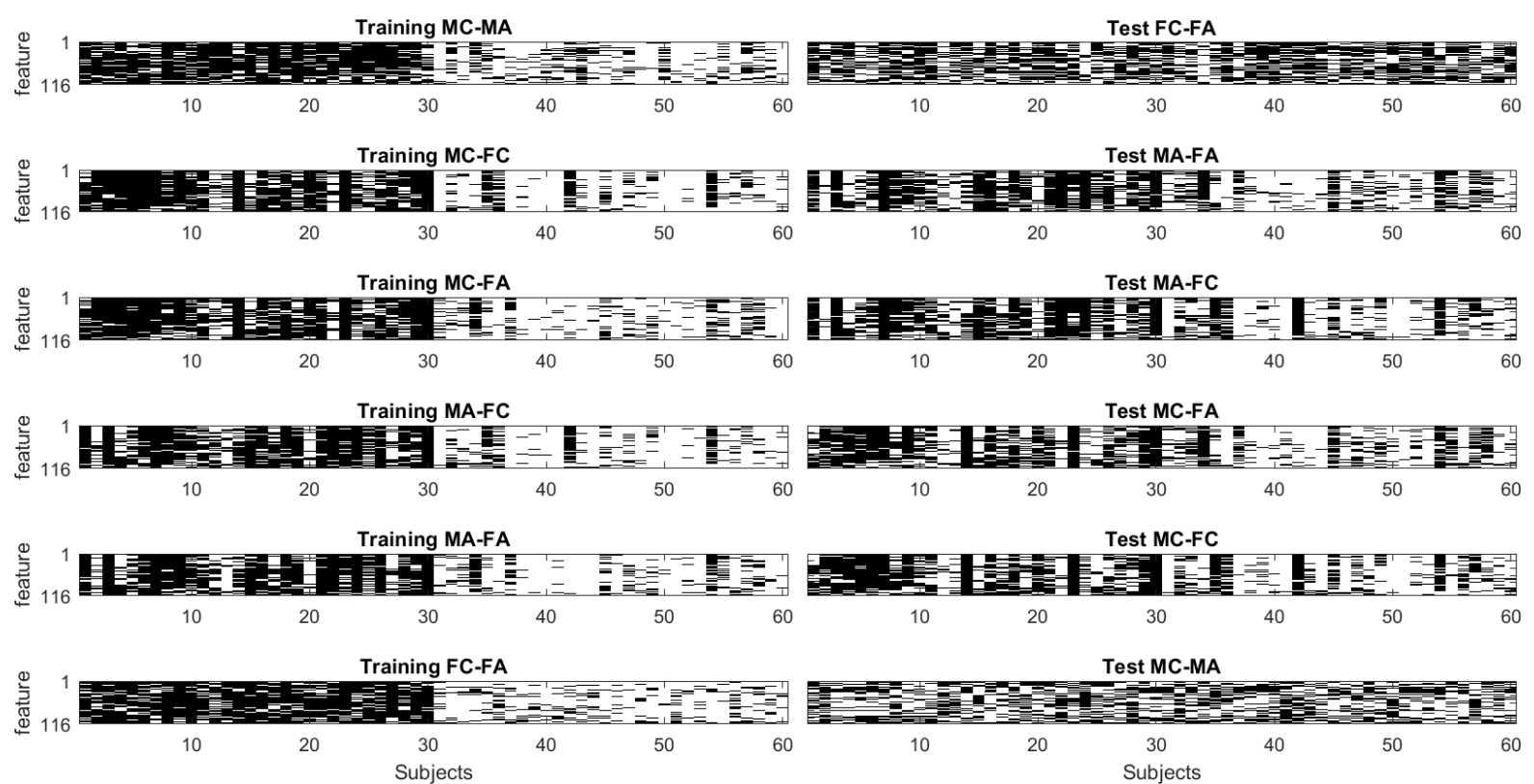

Fig. 7. Output score map of the linear SVM in the training\&test configuration (GM). On the left column, we plot the SVM scores obtained from the training set while on the right column, we show the consequent prediction on the test set. Each row contains one of the 6 binary problems to solve the multiclass framework, depicting all the 116 regionwise features ( $Y$-axis) versus each subject (X-axis). The hyperplane derived at each row using the training subsets on the left is employed in the prediction of the test subsets on the right. Note that for the ideal linearly-separable problem, we would obtain two black (from 1 to 30) and white (from 31 to 60) columns in all cases. In this way, the fitted learner should generalize fairly well on the remaining groups, obtaining two additional black and white columns on the test set, in case the features on the right were characterizing the groups on the left. This is clearly not the case in the 1 and 6 rows, where we are trying to predict male/female autistic features from the opposite sex.

Table 2. GM (WM) overlap and region-averaged accuracy of the output score maps (\%) for the selected subsets acting as training and test sets (Groups 1 and 6 are not considered).

\begin{tabular}{lccccc}
\hline \multirow{2}{*}{ Subsets } & $\begin{array}{c}\text { Train } \\
\text { Ov. }\end{array}$ & $\begin{array}{c}\text { Test } \\
\text { Ov. }\end{array}$ & Train Acc. & Test Acc. & \\
\hline MC & 90.98 & 89.68 & $77.07(7.68)$ & $63.03(9.05)$ & \\
FC & 92.16 & 90.43 & $80.60(6.65)$ & $70.88(7.84)$ & $\Omega$ \\
MA & 92.27 & 89.34 & $73.09(7.96)$ & $64.73(7.28)$ & Z \\
FA & 90.55 & 90.00 & $81.16(6.33)$ & $69.47(8.19)$ & \\
MC & 90.92 & 86.95 & $81.38(9.16)$ & $67.30(9.98)$ & \\
FC & 91.18 & 88.97 & $80.00(8.58)$ & $70.52(9.23)$ & \\
MA & 93.22 & 87.64 & $77.63(9.00)$ & $64.74(8.50)$ & L \\
FA & 91.24 & 87.82 & $80.45(8.88)$ & $66.16(9.39)$ & \\
\hline
\end{tabular}

Note: The GM overlap of correctly classified patterns in the MC subset, used at the training stage in the comparisons $\mathrm{MC}$ versus $\mathrm{FC}$ and $\mathrm{MC}$ versus $\mathrm{FA}$, is $90.98 \%$.

respect to the controlled upper bound (around 10\%), excluding the $G 1$ and $G 6$ which behave as random classifiers on the test set. This effect indicates the varying nature in the differences between $\mathrm{MC}$ and
MA (male autism features) which cannot be extrapolated to the FC-FA comparison and vice versa. In the former groups, the gender feature is the most prevalent, providing a high overlap in the output of the systems for several comparisons, see Table 2.

Participants with a high or low probability of being classified as having autism may be determined by using a predictive mapping approach ${ }^{37}$ Given the output score map of a participant with sex $S$ and condition $C$ at the test\&training stages, we first calculated the predictive class probability with respect to the male typical neurophenotype. For this purpose, the ratio was obtained of the number of regions associated with a classification of "male" to the overall number of brain regions. Then, we collected all these probabilities, one for each individual, into a discrete set of bins from 0 to 1 in steps of 0.25 , and computed the sample Autism probability (that is, the probability of the male neurophenotype, denoted in Fig. 9 as $P(p h=M \mid S, C=\mathrm{ASD}))$ as the ratio of the number of individuals classified with autism to the total number of individuals within the bin. As 

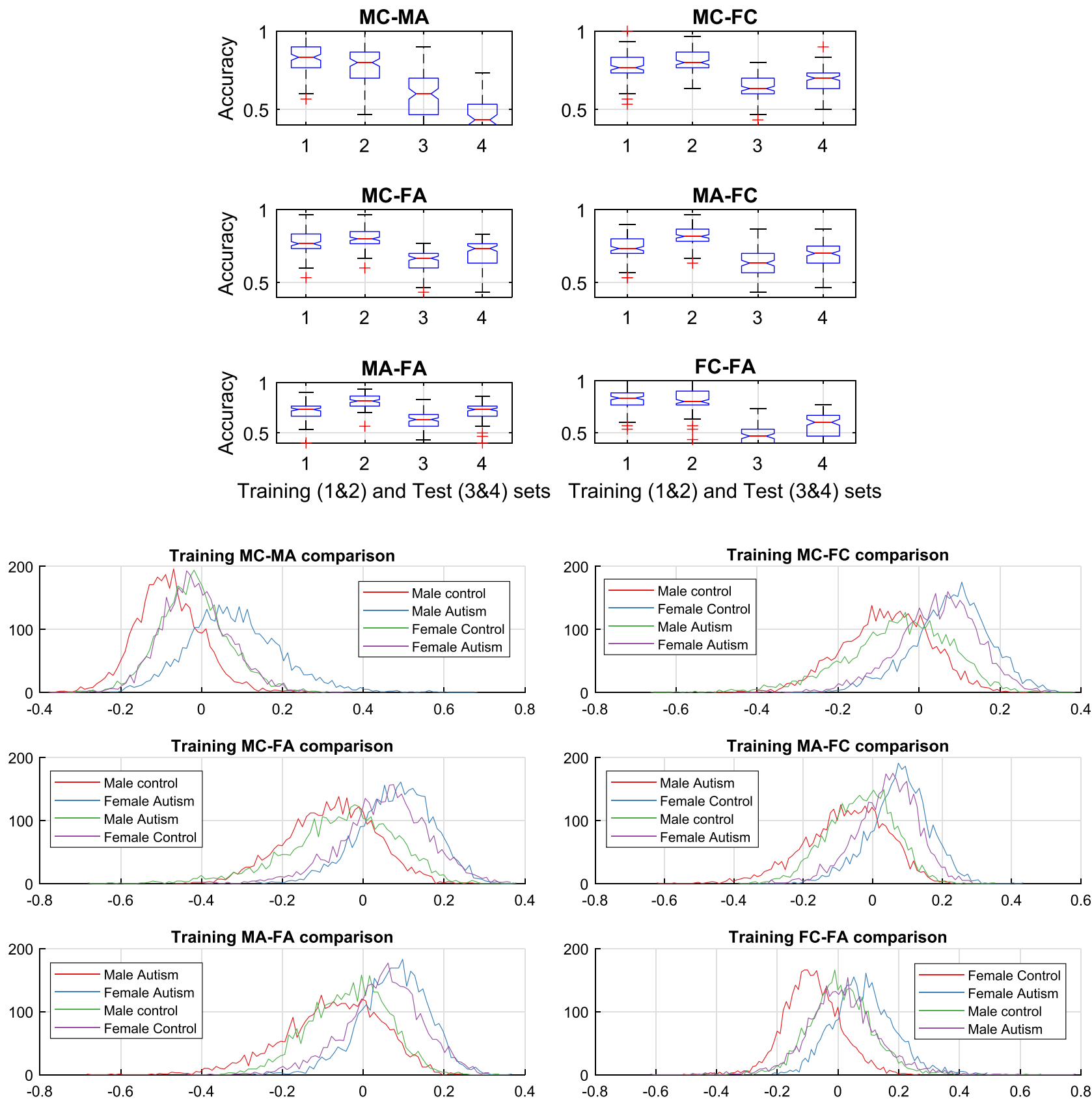

Fig. 8. Up: Distribution of accuracies in training and test subsets for Groups 1-6 (GM). Bottom: Overlay histogram analysis for Groups 1-6 (GM).

shown in Fig. 9 for $62-5$, where confidence intervals are determined using the Clopper-Pearson method in the binomial test,, 72 the probability of an individual being classified as autistic in males (blue) and females (red) evolves differently across the maleneurophenotypic axis. In addition, within gender, this probability increases or decreases depending on whether the group is processed at the test or training stage. This difference in behavior is due to the presence (or absence) of the autism feature that adds to the gender-based difference between groups, during the training stage.

In addition, we studied the binary classification problem using the group comparisons (MC-MA and 

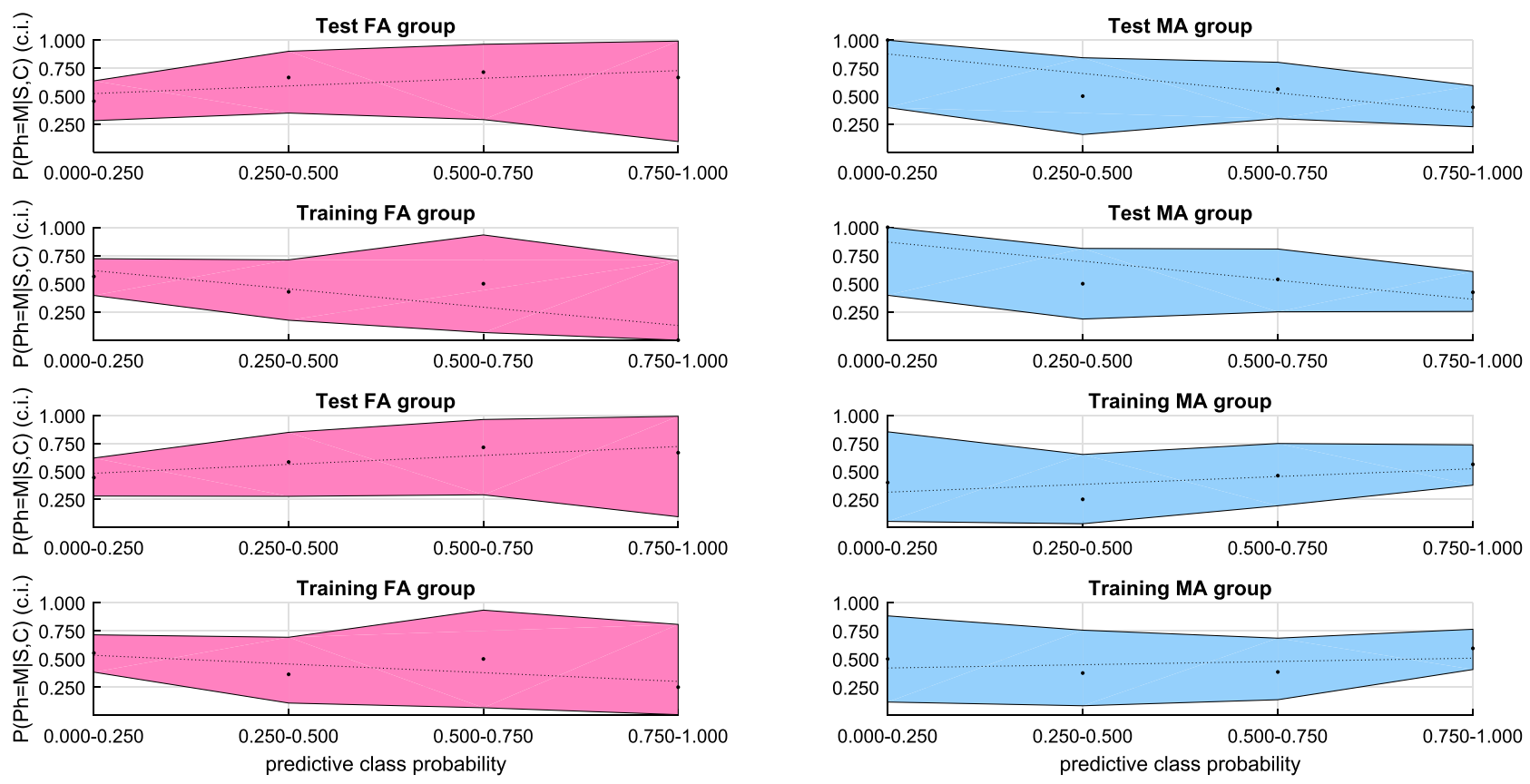

Fig. 9. (Color online) Probability for autism as a function of normative sex-related phenotypic variability in brain morphology: Probability estimates for autism across four discrete bins along the axis of predictive class probabilities for sex are plotted for $G 2-5$ and training $(T)$ and test $(t)$ stages. For male (blue) and female (red) models, the probability for autism behaves in a different manner with increasingly male-typical class predictions, that is, it increases (decreases) in females (males) at the test stage and contrarily at the training stage.

FC-FA) using a classical cross-validation scheme used in small sample sizes, i.e. leave-one-out cross-validation and $l=60$. In this case, we employed the same feature selection and extraction methods with a similar parameter tuning prior to linear SVMbased classification. As an example, for the MC-MA comparison, the few regions extracted on GM by this more restrictive cross-validation scheme are coinci-

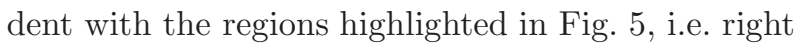
middle frontal gyrus, right pallidum, etc. yielding classification rates from $70 \%$ up to $80 \%$ in the study groups from GM and WM tissues.

Finally, using a one versus one decoding scheme based on a MAP strategy, the multiclass problem may be undertaken on a regionwise basis and a output score map of the linear SVM may be derived with four class levels. Under the upper bound $(\sim 0.1)$ on the actual risk for $d=1$ and using linear decision functions, the six binary classifiers can be combined obtaining the results shown in Fig. 10 Note in the upper subfigure that individuals with autism are ordered from male to female, and a deep (light) color represents control (autism) participant sex.
A regionwise analysis using this decoding strategy with GM reveals regions with a high accuracy (up to $83.33 \%$ ), namely, left and right parietal, temporal, occipital lobes, calcarine sulcus, etc. and a mean across region of $62.61 \pm 8.23$ with confusion depicted in the bottom of the same figure. With $\mathrm{WM}$, an improvement in performance is observed (up to $93.33 \%$ ) mainly due to the improved classification of the MC group (averaged accuracy across regions of $67.50 \pm 9.99$ ), with relevant regions located at middle frontal gyrus, inferior parietal lobe, cerebellar cortical crus II, middle occipital and temporal lobes, etc. Additionally, in this multiclass problem, we employed the output score maps depicted in Fig. 7 to decode the MAP output class for each region and then a majority voting scheme across the regions was applied to determine the final output class for each individual. Overall, the four groups are represented by different image patterns that can be classified with an accuracy up to $86.7 \%$ (91.70\% on WM) using the information contained in the entirety of the image, as shown in the confusion matrix (Fig. 111). 

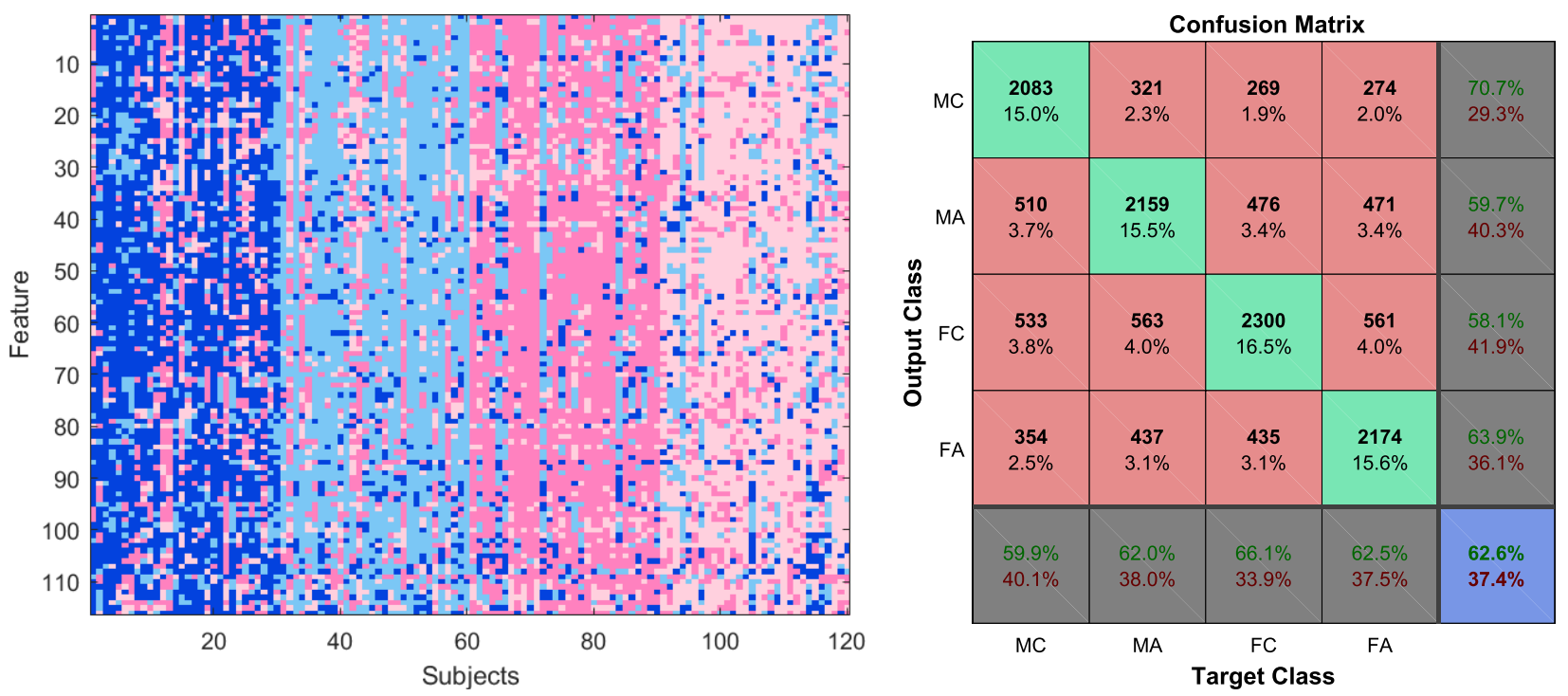

Fig. 10. Diagram on the left: Output score map of the combination of six binary classifiers in a one vs. one classification scheme using GM-based features. Right: Confusion matrix of the regionwise classification scheme.

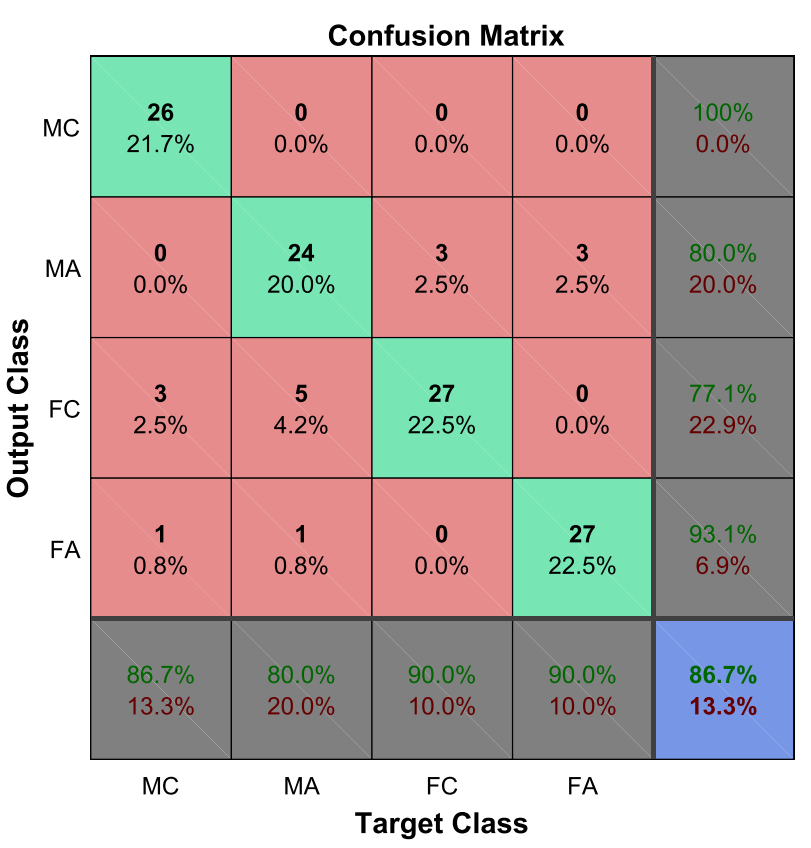

Fig. 11. Confusion matrix of the majority voting multiclass classification scheme corresponding to the output score map in Fig. 10. Observe how the overall classification result outperforms the regionwise approach, due to the presence of outliers in the four-class problem (patients containing a high ratio of misclassified regions).

\subsection{Permutation tests}

Although the experimental conditions were rigorously established in the previous sections, that is, the results obtained by linear classifiers in low
Table 3. Testing for significance the classification models on GM features (95\% significance level). Accepted regions in \% out of the 116 analyzed BAs for the binary classifiers at the training stage.

\begin{tabular}{cccccc}
\hline$\%$ & $\mathrm{MC}$ & $\mathrm{MA}$ & $\mathrm{FC}$ & $\mathrm{FA}$ & \\
\hline$G 1$ & 95.69 & 83.62 & - & - & \\
$G 2$ & 89.66 & - & 96.55 & - & \\
$G 3$ & 90.52 & - & - & 98.28 & Z \\
$G 4$ & - & 82.76 & 98.28 & & \\
$G 5$ & - & 80.17 & - & 98.28 & \\
$G 6$ & - & - & 91.38 & 94.83 & \\
\hline
\end{tabular}

dimensional spaces are theoretically meaningful at a significance level $\eta$ (see Sec. 3.1), a permutation test to validate these results was applied to the whole dataset. For the complete set of binary classifiers, the cross-validation procedure was repeated $n=500$ times after randomly permuting the class labels to derive $p$-value maps. As expected, the classification obtained with this analysis for the set of classifiers is almost random and, at the $95 \%$ level of confidence, almost all the regions were considered as significant in this analysis as shown in Table 3 The result of this analysis allows the construction of relevance maps for each classification model such as those displayed in Fig. 12, where, as an example, we illustrate the prediction of sex based on VBM features using the sex classifier for $G 2$. 


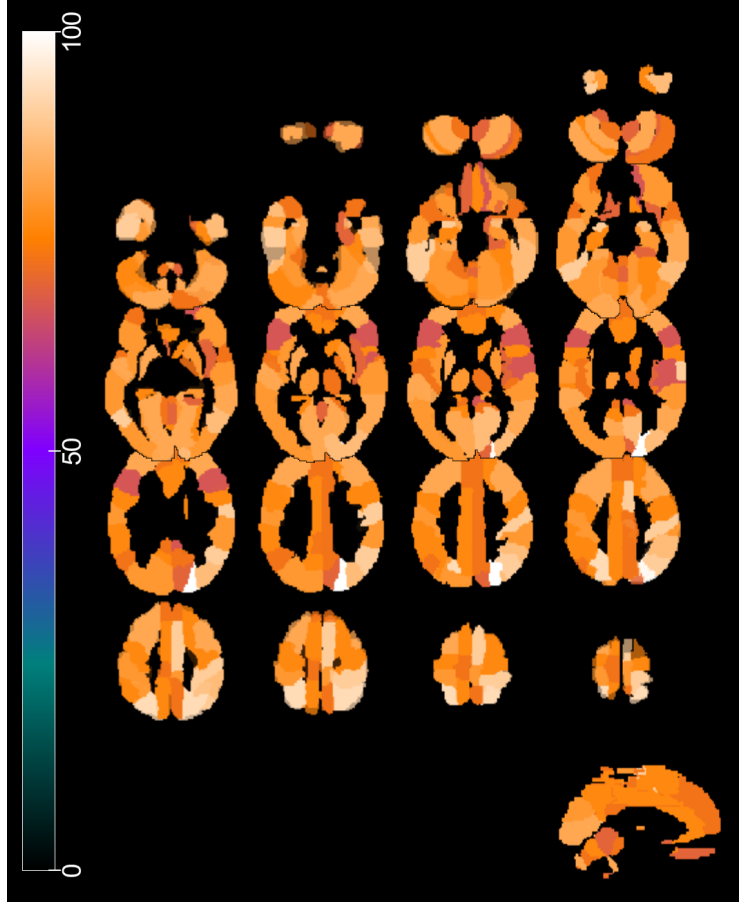

(a) MC relevant regions corrected by the permutation test

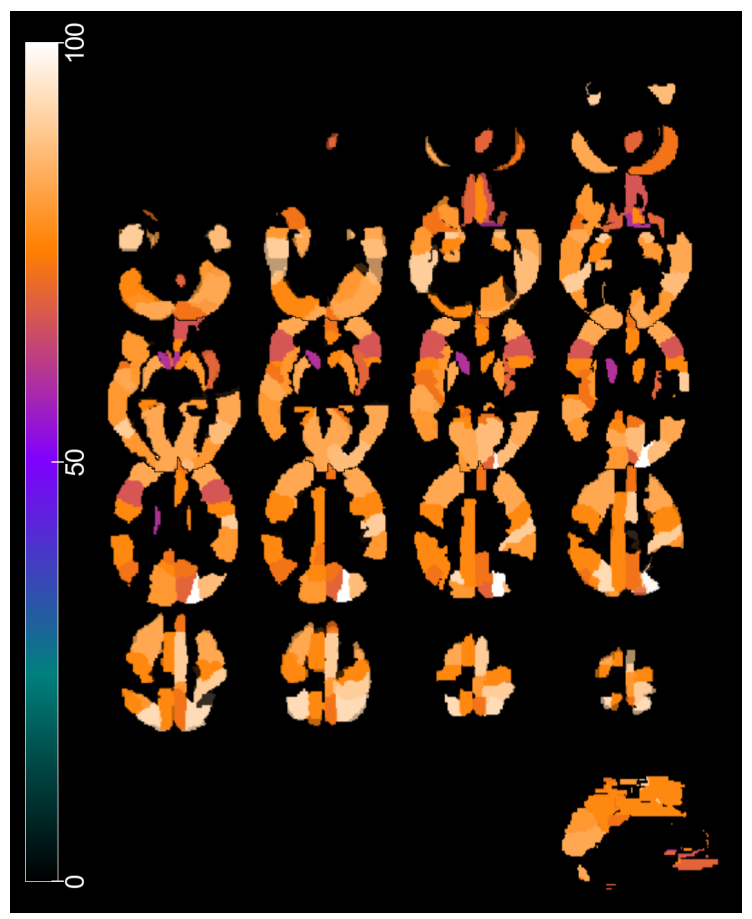

(c) MA relevant regions corrected by the permutation test

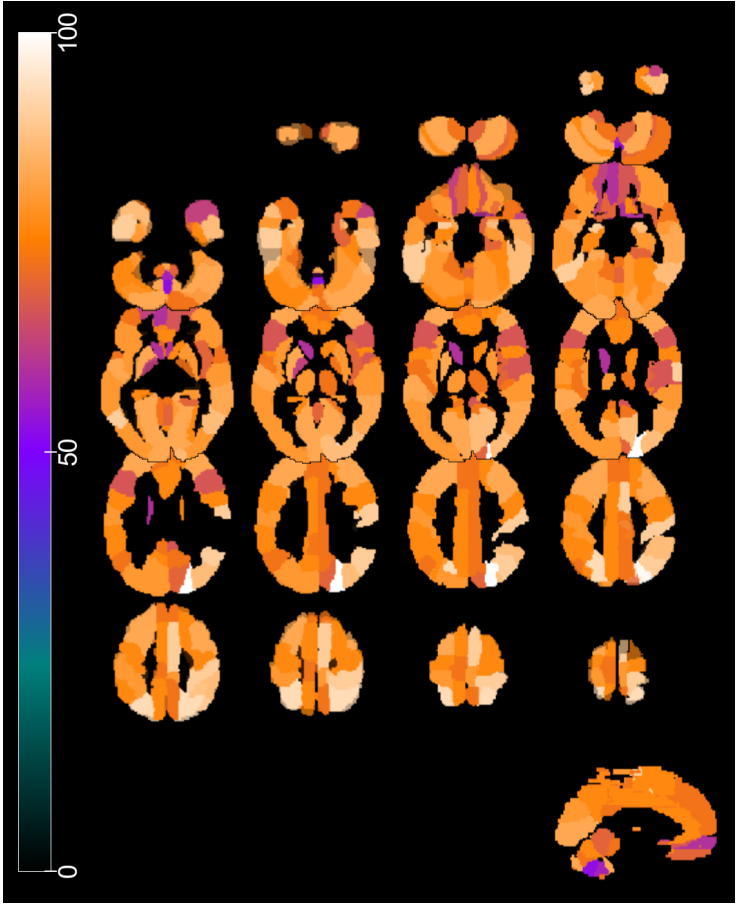

(b) FC relevant regions corrected by the permutation test

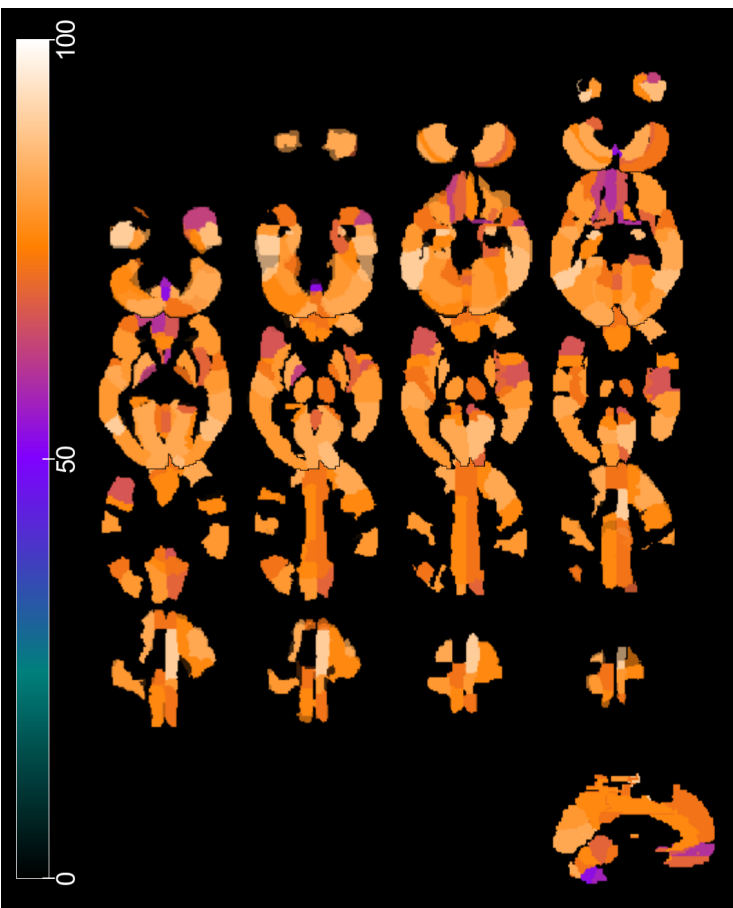

(d) FA relevant regions corrected by the permutation test

Fig. 12. (Color online) Accuracy maps for G2: Training MC-FC comparison and test on MA, FA groups with GM features. The colorbar indicates the precision of each region in the binary classification problem of detecting sexual dimorphism in the training and test subsets. 


\section{Discussion}

From the perspective of this machine learning-based approach, the neuroanatomy of autism in males and females is comprised of separate distinguishing patterns. A distinguishing pattern similarly identifies male and female controls. These group differences are observed even with a small sample set, although some theoretical bounds and suitable methodologies must be imposed to highlight such differences. The interactions between sex and diagnosis observed in this paper extend those previously found in prior work ${ }^{3437}$ that also showed sex $\times$ diagnosis group differences on GM and WM tissues. In particular, greater accuracy rates were obtained with the classification apparatus described herein.

In addition, based on this multivariate methodology, we also found that there was a significant overlap between the neuroanatomical features of autism in males and females, unlike the previous approaches, aligning with the predictions of the EMB theory in terms of the directionality of effect, and with the "gender incoherence" hypothesi ${ }^{74}$ ). These effects were observed by the use of a regionwise PLS-based activation map transformed into the same parameters as those used in the one-tailed $t$ maps derived from the group differences. In fact, the approach proposed in this paper considers not only the EMB theory ${ }^{71}$ directionality, but also its inverse, that is, predicting that males with autism are feminized in terms of neuroanatomy! This effect was clearly observed on GM and WM VBM comparisons and agrees with the results obtained by testing how the effect of autism overlaps with the effect of "feminization" using one-tailed contrasts. ${ }^{[44}$ Thus, on balance, these results may be a demonstration of gender incoherence of males with autism rather than "masculinization".

The heterogeneity of autism was detected by the assessment of the output score maps derived from the machine learning theory. The use of crossvalidation groups further reduced the limited sample size and degraded the performance of the CAD system. Although some considerations and preventive measures regarding the curse of dimensionality were carried out to be conservative, the observed effects and group differences could be partially samplespecific and the current analysis requires replication on larger datasets. These difficulties are detected in terms of the presence of outliers, that is, participants that are misclassified by resubstitution in almost all the analyzed regions. Those participants that are located in the "wrong" feature subspace present an heterogeneous pattern that affects the performance of the SVM when they are considered in any validation/training fold. The output score maps revealed this misclassified sex/condition-specific neurophenotype on GM and WM tissues across regions and participants.

\section{Conclusions}

In summary, the research developed here is a first attempt to describe both sex-typical multivariate neurobiological phenotypes by the use of machine learning and an MRI sample of equal-sized highfunctioning male and female adults with and without autism. Although the main limitation of the current equal-sized gender datasets is the samples sizes, we avoided this difficulty by imposing some restrictions on the learning parameters of the system by the use of a novel upper bound in the resubstitution estimate, obtaining a good trade-off between empirical risk and the variance of the actual risk estimation (upper bound). In addition, the system used a set of features extracted from PLS activation maps that are demonstrated to be statistically significant and in accordance with two theories of the neurobiology of autism. The complete classification system in a one versus one configuration achieves, under these theoretical conditions, high classification results (up to $86 \%$ ) in a four-label classification problem, thus outperforming the classification rate of the previous published works on this field. 34,75 , 77

\section{Acknowledgments}

This work was partly supported by the MINECO Under the TEC2015-64718-R Project, the Salvador de Madariaga Mobility Grants 2017 and the Consejería de Economía, Innovación, Ciencia y Empleo (Junta de Andalucía, Spain) under the Excellence Project P11-TIC-7103. The study was conducted in association with the National Institute for Health Research Collaborations for Leadership in Applied Health Research and Care (NIHR CLAHRC) East of England (EoE). The Project was supported by the UK Medical Research Council (Grant No. GO 400061) and European Autism Interventions - a Multicentre Study for Developing New Medications 
(EU-AIMS); EU-AIMS has received support from the Innovative Medicines Initiative Joint Undertaking Under Grant Agreement No. 115300, resources of which are composed of financial contribution from the European Union's Seventh Framework Programme (FP7/2007-2013) and EFPIA companies' in-kind contribution. During the period of this work, M-CL was supported by the OBrien Scholars Program in the Child and Youth Mental Health Collaborative at the Centre for Addiction and Mental Health (CAMH) and The Hospital for Sick Children, Toronto, the Academic Scholar Award from the Department of Psychiatry, University of Toronto, the Slaight Family Child and Youth Mental Health Innovation Fund, CAMH Foundation, and the Ontario Brain Institute via the Province of Ontario Neurodevelopmental Disorders (POND) Network; MVL was supported by the British Academy, Jesus College Cambridge, Wellcome Trust, and an ERC Starting Grant (ERC-2017-STG; 755816); SB-C was supported by the Autism Research Trust. The views expressed are those of the authors and not necessarily those of the NHS, the NIHR or the Department of Health, UK.

\section{Appendix A}

\section{A.1. On the upper bound of the actual risk}

In terms of the one-sided uniform convergence of the means, we are interested in assessing for a given significance level $\eta$ :

$$
P\left\{\sup _{i}\left(P\left(\alpha_{i}\right)-P_{\mathrm{emp}}\left(\alpha_{i}\right)\right)>\epsilon\right\}<\eta,
$$

where $P\left(\alpha_{i}\right)=P\left(F\left(x, \alpha_{i}\right)\right)$. Of course, with a sample size $l \rightarrow \infty$, the law of large numbers expressed in terms of the third Hoeffding inequality ${ }^{59}$ for any functional $\alpha_{i}$ establishes that

$$
\lim _{l \rightarrow \infty} P\left\{\sup _{i}\left(P\left(\alpha_{i}\right)-P_{\mathrm{emp}}\left(\alpha_{i}\right)\right)>\epsilon\right\}=0
$$

and the uniform convergence in Eq. (A.1) is achieved. In other cases $l<\infty$, the aforementioned inequality can be used to establish the bound of the actual risk as $\sqrt{57}$

$$
P\left\{\Gamma_{i}>\epsilon\right\} \leq \sum_{i=1}^{N} P\left\{\gamma_{i}>\epsilon\right\}<\eta=N \exp \left(-2 \epsilon^{2} l\right),
$$

where $\Gamma_{i}=\sup _{i}\left(\gamma_{i}\right)$ and $\gamma_{i}=P\left(\alpha_{i}\right)-P_{\mathrm{emp}}\left(\alpha_{i}\right)$ and $N$ is the finite number of functional dependencies. Since the inequality is valid for all decision functions $F\left(x, \alpha_{i}\right)$, the actual risk obtained by $\alpha_{\mathrm{emp}}$ is bounded with probability $1-\eta$ by

$$
\gamma_{\mathrm{emp}} \leq \sqrt{\frac{1}{2 l} \ln \left(\frac{N}{\eta}\right)}
$$

that is equivalent to Eq. (3). In general, these bounds could be further improved by considering the relative deviations ${ }^{57 / 60}$ under scenarios, where $P\left(\alpha_{i}\right)$ tends to the extremes 0,1 , but that is far from our problem.

Definition. A set of $l$ vectors is in general position in $d$-space if every subset of $d$ or fewer vectors is linearly independent.

Consider that the training sample $\left\{\mathbf{x}_{i}, y_{i}\right\}$ is distributed randomly in general position, then the number of linearly separable dichotomies of the set of input patterns is equal to $N$, that is, the number of decision functions $F(\mathbf{x}, \alpha)$ when the training sample is not a root of the set $\left(F\left(\mathbf{x}_{i}, \alpha\right) \neq 0\right)$. As shown in Ref. 55 for linear decision functions and based on the Function Counting Theorem, this is equal to

$$
N(l, d)=2 \sum_{k=0}^{d-1}\left(\begin{array}{c}
l-1 \\
k
\end{array}\right) .
$$

Thus, the bound in Eq. (3) could be rewritten as shown in Eq. (44). As an example, if $d \simeq l$, then $N(l, d) \simeq 2^{l}$, thus the number of functions is such that it separates the sample size in all possible ways (nonfalsifiable learning machine), the minimum of the empirical risk is zero and the upper bound of $P\left(\alpha_{\mathrm{emp}}\right)$ is trivial $(>0.5)$ independently of the sample size $l$. On the contrary, if $d \ll l$, the actual risk reaches its maximum value close to the empirical risk, i.e. for $d=\{1,2,3\}$ and $l=120$, the maximum deviations of the frequencies $\left(\Delta P=P-P_{\mathrm{emp}}\right)$ are obtained with probability $1-\eta(=0.95)$ as

$$
\Delta P \leq\{0.1398,0.1879,0.2286\} .
$$

\section{Appendix B}

The MRC AIMS Consortium is a UK collaboration between the Institute of Psychiatry, Psychology and Neuroscience (IoPPN) at Kings College, London, the Autism Research Centre, University of Cambridge, and the Autism Research Group, University of Oxford. The Consortium members are 
in alphabetical order: Anthony J. Bailey (Oxford), Simon Baron-Cohen (Cambridge), Patrick F. Bolton (IoPPN), Edward T. Bullmore (Cambridge), Sarah Carrington (Oxford), Marco Catani (IoPPN), Bhismadev Chakrabarti (Cambridge), Michael C. Craig (IoPPN), Eileen M. Daly (IoPPN), Sean C. L. Deoni (IoPPN), Christine Ecker (IoPPN), Francesca Happé (IoPPN), Julian Henty (Cambridge), Peter Jezzard (Oxford), Patrick Johnston (IoPPN), Derek K. Jones (IoPPN), Meng-Chuan Lai (Cambridge), Michael V. Lombardo (Cambridge), Anya Madden (IoPPN), Diane Mullins (IoPPN), Clodagh M. Murphy (IoPPN), Declan G. M. Murphy (IoPPN), Greg Pasco (Cambridge), Amber N. V. Ruigrok (Cambridge), Susan A. Sadek (Cambridge), Debbie Spain (IoPPN), Rose Stewart (Oxford), John Suckling (Cambridge), Sally J. Wheelwright (Cambridge), and Steven C. Williams (IoPPN).

\section{References}

1. J. Piven, S. Arndt, J. Bailey, S. Havercamp, N. C. Andreasen and P. Palmer, An MRI study of brain size in autism, Am. J. Psychiatry 152(8) (1995) 1145-1149.

2. E. Redcay and E. Courchesne, When is the brain enlarged in autism? A meta-analysis of all brain size reports, Biol. Psychiatry 58(1) (2005) 1-9.

3. J. Radua, E. Via, M. Catani and D. Mataix-Cols, Voxel-based meta-analysis of regional white-matter volume differences in autism spectrum disorder versus healthy controls, Psychol. Med. 41(7) (2011) 1539-1550.

4. N. Traut, A. Beggiato, T. Bourgeron, R. Delorme, L. Rondi-Reig, A. L. Paradis and R. Toro, Cerebellar volume in autism: Literature meta-analysis and analysis of the autism brain imaging data exchange cohort, Biol. Psychiatry 83(7) (2018) 579-588.

5. R. Sacco, S. Gabriele and A. M. Persico, Head circumference and brain size in autism spectrum disorder: A systematic review and meta-analysis, Psychiatry Res. 234(2) (2015) 239-251.

6. L. D. Eggert, J. Sommer, A. Jansen, T. Kircher and C. Konrad, Accuracy and reliability of automated gray matter segmentation pathways on real and simulated structural magnetic resonance images of the human brain, PLoS One 7(9) (2012) e45081.

7. C. Scarpazza, S. Tognin, S. Frisciata, G. Sartori and A, Mechelli, False positive rates in Voxel-based morphometry studies of the human brain: Should we be worried? Neurosci. Biobehav. Rev. 52 (2015) 49-55.

8. D. C. Glahn, A. R. Laird, I. Ellison-Wright, S. M. Thelen, J. L. Robinson, J. L. Lancaster, E. Bullmore and P. T. Fox, Meta-analysis of gray matter anomalies in schizophrenia: Application of anatomic likelihood estimation and network analysis, Biol. Psychiatry 64(9) (2008) 774-781.

9. C. H. Lai, Gray matter volume in major depressive disorder: A meta-analysis of voxel-based morphometry studies, Psychiatry Res. 211(1) (2013) 37-46.

10. E. Bora, A. Fornito, C. Pantelis and M. Yucel, Gray matter abnormalities in major depressive disorder: A meta-analysis of voxel based morphometry studies, J. Affect. Disord. 138(1-2) (2012) 9-18.

11. T. Nickl-Jockschat, U. Habel, T. M. Michel, J. Manning, A. R. Laird, P. T. Fox, F. Schneider and S. B. Eickhoff, Brain structure anomalies in autism spectrum disorder - A meta-analysis of VBM studies using anatomic likelihood estimation, Hum. Brain Mapp. 33(6) (2012) 1470-1489.

12. F. Cauda, T. Costa, S. Palermo, F. D'Agata, M. Diano, F. Bianco, S. Duca and R. Keller, Concordance of white matter and gray matter abnormalities in autism spectrum disorders: A voxel-based metaanalysis study, Hum. Brain Mapp. 35(5) (2014) 2073-2098.

13. K. K. Yu, C. Cheung, S. E. Chua, G. M. McAlonan, Can Asperger syndrome be distinguished from autism? An anatomic likelihood meta-analysis of MRI studies, J. Psychiatry Neurosci. 36(6) (2011) 412-421.

14. K. J. Worsley, S. Marrett, P. Neelin, A. C. Vandal, K. J. Friston and A. C. Evans, A unified statistical approach for determining significant signals in images of cerebral activation, Hum. Brain Mapp. 4(1) (1996) 58-73.

15. D. C. Rojas, E. Peterson, E. Winterrowd, M. L. Reite, S. J. Rogers and J. R. Tregellas, Regional gray matter volumetric changes in autism associated with social and repetitive behavior symptoms, BMC Psychiatry 6 (2006) 56.

16. T. P. DeRamus and R. K. Kana, Anatomical likelihood estimation meta-analysis of gray and white matter anomalies in autism spectrum disorders, Neuroimage, Clin. 7 (2014) 525-536.

17. X. Yang, T. Si, Q. Gong, L. Qiu, Z. Jia, M. Zhou, Y. Zhao, X. Hu, M. Wu and H. Zhu, Brain gray matter alterations and associated demographic profiles in adults with autism spectrum disorder: A metaanalysis of voxel-based morphometry studies, Aust. N. Z. J. Psychiatry 50(8) (2016) 741-753.

18. C. O. Carlisi, L. J. Norman, S. S. Lukito, J. Radua, D. Mataix-Cols and K. Rubia, Comparative multimodal meta-analysis of structural and functional brain abnormalities in autism spectrum disorder and obsessive-compulsive disorder, Biol. Psychiatry 82(2) (2017) 83-102.

19. R. Chen, Y. Jiao and E. H. Herskovits, Structural MRI in autism spectrum disorder, Pediatr. Res. 69(5 Pt 2) (2011) 63R-8R. 
20. R. K. Lenroot and P. K. Yeung, Heterogeneity within autism spectrum disorders: What have we learned from neuroimaging studies? Front. Hum. Neurosci. 7 (2013) 733.

21. C. Lord and R. M. Jones, Annual research review: Re-thinking the classification of autism spectrum disorders, J. Child Psychol. Psychiatry 53(5) (2012) 490-509.

22. Diagnostic and Statistical Manual of Mental Disorders, 5th edn. (American Psychiatric Association, Arlington, 2013), https://doi.org/10.1176/appi. books.9780890425596.

23. L. Waterhouse and C. Gillberg, Why autism must be taken apart, J. Autism Dev. Disord. 44(7) (2014) 1788-1792.

24. C. W. Nordahl, N. Lange, D. D. Li, L. A. Barnett, A. Lee, M. H. Buonocore, T. J. Simon, S. Rogers, S. Ozonoff and D. G. Amarala, Brain enlargement is associated with regression in preschool-age boys with autism spectrum disorders, Proc. Natl. Acad. Sci. USA 108(50) (2011) 20195-20200.

25. S. Haar, S. Berman, M. Behrmann and I. Dinstein, Anatomical abnormalities in autism?, Cereb. Cortex. 26(4) (2016) 1440-1452.

26. K. Riddle, C. J. Cascio and N. D. Woodward, Brain structure in autism: A voxel-based morphometry analysis of the Autism Brain Imaging Database Exchange (ABIDE), Brain Imaging Behav. 11(2) (2017) 541-551.

27. F. D. Beacher, L. Minati, S. Baron-Cohen, M. V. Lombardo, M. C. Lai, M. A. Gray, N. A. Harrison and H. D. Critchley, Autism attenuates sex differences in brain structure: A combined voxel-based morphometry and diffusion tensor imaging study, Am. J. Neuroradiol 33(1) (2012) 83-89.

28. L. Q. Uddin, V. Menon, C. B. Young, S. Ryali, T. Chen, A. Khouzam, N. J. Minshew and A. Y. Hardan, Multivariate searchlight classification of structural magnetic resonance imaging in children and adolescents with autism, Biol. Psychiatry 70(9) (2011) 833-841.

29. S. Baron-Cohen, F. J. Scott, C. Allison, J. Williams, P. Bolton, F. E. Matthews et al., Prevalence of autism-spectrum conditions: UK school-based population study, Br. J. Psychiatry 194 (2009) 500-509.

30. M. L. Mattila, M. Kielinen, S. L. Linna, K. Jussila, H. Ebeling, R. Bloigu et al., Autism spectrum disorders according to DSM-IV-TR and comparison with DSM-5 draft criteria: An epidemiological study, J. Am. Acad. Child. Adolesc. Psychiatry 50 (2011) 583-592.

31. S. Idring, D. Rai, H. Dal, C. Dalman, H. Sturm, E. Zander et al., Autism spectrum disorders in the stockholm youth cohort: Design, prevalence and validity, PLoS One 7 (2012) e41280.

32. Y. S. Kim, B. L. Leventhal, Y. J. Koh, E. Fombonne, E. Laska, E. C. Lim et al., Prevalence of autism spectrum disorders in a total population sample, Am. J. Psychiatry 168 (2011) 904-912.

33. E. Via, J. Radua, N. Cardoner, F. Happe and D. Mataix-Cols, Meta-analysis of gray matter abnormalities in autism spectrum disorder: Should Asperger disorder be subsumed under a broader umbrella of autistic spectrum disorder? Arch. Gen. Psychiatry 68 (2011) 409-418.

34. M. C. Lai, M. V. Lombardo, J. Suckling, A. N. V. Ruigrok, B. Chakrabarti, C. Ecker, S. C. Deoni, L. Craig, M. C. D. G. M. Murphy, E. T. Bullmore and S. Baron-Cohen, Biological sex affects the neurobiology of autism, Brain 136(9) (2013) 2799-2815.

35. M. C. Lai, M. V. Lombardo, B. Chakrabarti, S. A. Sadek, G. Pasco, S. J. Wheelwright, E. T. Bullmore and S. Baron-Cohen, M. R. C. AIMS Consortium and J. Suckling, A shift to randomness of brain oscillations in people with autism, Biol. Psychiatry 68(12) (2010) 1092-1099.

36. M. V. Lombardo, B. Chakrabarti, E. T. Bullmore, S. A. Sadek, G. Pasco, S. J. Wheelwright et al., Atypical neural self-representation in autism, Brain 133(Pt 2) (2010) 611-624.

37. C. Ecker, C. Ginestet, Y. Feng, P. Johnston, M. V. Lombardo, M. C. Lai, J. Suckling, L. Palaniyappan, E. Daly, C. M. Murphy, S. C. Williams, E. T. Bullmore, S. Baron-Cohen, M. Brammer and D. G. Murphy, Brain surface anatomy in adults with autism: The relationship between surface area, cortical thickness, and autistic symptoms, JAMA Psychiatry 70 (2013) 59-70.

38. C. Ecker, D. S. Andrews, C. M. Gudbrandsen et al., Association between the probability of autism spectrum disorder and normative sex-related phenotypic diversity in brain structure, JAMA Psychiatry 74(4) (2017) 329-338.

39. A. Ortiz, J. Munilla, I. Álvarez-Illán, J. M. Górriz and J. Ramírez, Exploratory graphical models of functional and structural connectivity patterns for Alzheimer's disease diagnosis, Front. Comput. Neurosci. 9 (2015) 132.

40. U. Acharya, R. Yanti, J. Zheng, M. Krishnan, J. Tan, R. Martis and C. Lim, Automated diagnosis of epilepsy using cwt, hos and texture parameters, Int. J. Neural Syst. 23(3) (2013) 1350009.

41. A. R. McIntosh, F. L. Bookstein, J. V. Haxby and C. L. Grady, Spatial pattern analysis of functional brain images using partial least squares, Neuroimage $\mathbf{3}(3$ Pt 1) (1996) 143-157.

42. A. Ortiz, J. M. Górriz, J. Ramírez and F. MartínezMurcia, Lvq-SVM based CAD tool applied to structural MRI for the diagnosis of the alzheimer's disease, Pattern Recognit. Lett. 34 (2013) 17251733 .

43. F. Martinez-Murcia, J. Górriz, J. Ramírez, M. Moreno-Caballero, M. Gómez-Río, P. P. M. Initiative et al., Parametrization of textural patterns in 
123i-ioflupane imaging for the automatic detection of parkinsonism, Med. Phys. 41(1) (2014) 012502.

44. C. Ecker, J. Suckling, S. C. Deoni, M. V. Lombardo, E. T. Bullmore, S. Baron-Cohen, M. Catani, P. Jezzard, A. Barnes, A. J. Bailey, S. C. Williams and D. G. M. Murphy, Brain anatomy and its relationship to behavior in adults with autism spectrum disorder a multicenter magnetic resonance imaging study, Arch. Gen. Psychiatry 69(2) (2012) 195-209, doi:10.1001/archgenpsychiatry.2011.1251.

45. D. Chyzhyk, M. Graña, D. Öngür and A. K. Shinn, Discrimination of schizophrenia auditory hallucinators by machine learning of resting-state functional MRI, Int. J. Neural Syst. 25 (2015) 1550007.

46. L. Ronan, R. Pienaar, G. Williams, E. Bullmore, T. J. Crow, N. Roberts, P. B. Jones, J. Suckling and P. C. Fletcher, Intrinsic curvature: A marker of milimeter-scale tangential cortico-cortical connectivity? Int. J. Neural Syst. 21(5) (2011) 351366.

47. M. Ahmadlou and H. Adeli, Functional community analysis of brain: A new approach for EEG-based investigation of the brain pathology, Neuroimage 58 (2011) 401-408.

48. I. A. Illan, J. M. Górriz, J. Ramírez and A. MeyerBase, Spatial component analysis of MRI data for alzheimer's disease diagnosis: A bayesian network approach, Front. Comput. Neurosci. 8 (2014) 156.

49. F. Segovia, J. M. Górriz, J. Ramírez, I. Alvarez, J. M. Jiménez-Hoyuela, Improved parkinsonism diagnosis using a partial least squares based approach, Med. Phys. 39(7) (2012) 4395-4403.

50. B. S. Khundrakpam, J. Tohka and A. C. Evans, Prediction of brain maturity based on cortical thickness at different spatial resolutions, NeuroImage 111 (2015) 350-359.

51. I. Guyon, J. Weston, S. Barnhill and V. Vapnik, Gene selection for cancer classification using support vector machines, Mach. Learn. 46(1) (2002) 389422.

52. J. Bi, K. Bennett, M. Embrechts, C. Breneman and M. Song, Dimensionality reduction via sparse support vector machines, J. Mach. Learn. Res. 3 (2003) 1229-1243.

53. E. Parrado-Hernandez, V. Gomez-Verdejo, M. Martinez-Ramon, J. Shawe-Taylor, P. Alonso, J. Pujol, J. M. Menchon, N. Cardoner and C. SorianoMas, Discovering brain regions relevant to obsessive compulsive disorder identification through bagging and transduction, Med. Image Anal. 18(3) (2014) 435-448.

54. J. M. Górriz, J. Ramírez, J. Suckling, I. Á. Illán, A. O. García, F. J. Martínez-Murcia, F. Segovia, D. Salas-Gonzalez and S. Wang, Case-based statistical learning: A non-parametric implementation with a conditional-error rate SVM, IEEE Access 5 (2017) $11468-11478$.
55. J. M. Gorriz, J. Ramirez, J. Suckling, F. J. Martinez-Murcia, I. A. Illán, F. Segovia, A. Ortiz, D. Salas-González and D. Castillo-Barnés and C. G. Puntonet, A semi-supervised learning approach for model selection based on classhypothesis testing, Expert Syst. Appl. 90 (2017) 4049, http://dx.doi.org/10.1016/j.eswa.2017.08.006.

56. S. Escalera, O. Pujol and P. Radeva, On the decoding process in ternary error-correcting output codes, IEEE Trans. Pattern Anal. Mach. Learn. Intell. 32(1) (2010) 120-134.

57. V. Vapnik, A method of minimizing empirical risk for the problem of pattern recognition, Estimation of Dependences Based on Empirical Data, Springer Series in Statistics (Springer-Verlag, New York, 1982), pp. 139-161.

58. V. N. Vapnik, The Nature of Statistical Learning Theory (Springer-Verlag, New York, 1995).

59. W. Hoeffding, Probability inequalities for sums of bounded random variables, J. Am. Stat. Assoc. 58 (1963) 13-30.

60. O. Gascuel and G. Caraux, Distribution-free performance bounds with the resubstitution error estimate, Pattern Recognit. Lett. 13(11) (1992) 757764.

61. T. M. Cover, Geometrical statistical and applications in inequalities linear of pattern properties recognition systems with applications in pattern recognition, IEEE Trans. Electron. Comput. 3 (1965) 326-334.

62. N. Tzourio-Mazoyer et al., Automated anatomical labeling of activations in SPM using a macroscopic anatomical parcellation of the MNI MRI singlesubject brain, NeuroImage 15(1) (2002) 73-279.

63. K. Friston, J. Ashburner, S. Kiebel, T. Nichols and W. Penny, Statistical Parametric Mapping (Academic Press, London, 2007).

64. C. Gaser and R. Dahnke, CAT - A computational anatomy toolbox for the analysis of structural MRI data, HBM 2016, http://www.neuro.unijena.de/hbm2016/GaserHBM2016.pdf (2016).

65. J. Ashburner, A fast diffeomorphic image registration algorithm, NeuroImage 38(1) (2007) 95-113, https://doi.org/10.1016/j.neuroimage.2007.07.007.

66. A. Shiino, T. Watanabe, K. Maeda, E. Kotani, I. Akiguchi and M. Matsuda, Four subgroups of Alzheimer's disease based on patterns of atrophy using VBM and a unique pattern for early onset disease, NeuroImage 33 (2006) 17-26.

67. N. Ayache, Analyzing 3D Images of the Brain, NeuroImage 4(3) (1996) S34-S35.

68. I. Guyon, S. Gunn, M. Nikravesh and L. A. Zadeh (eds.), Feature Extraction: Foundations and Applications (Springer, Berlin, 2006).

69. R. Rosipal and N. Kramer, Overview and Recent Advances in Partial Least Squares (Springer Berlin, Heidelberg, 2006), pp. 34-51. 
70. M. Hazewinkel (ed.), Student Distribution, Encyclopedia of Mathematics (Springer Science+Business Media B.V./Kluwer Academic Publishers, Berlin, 2001).

71. S. Baron-Cohen, The extreme male brain theory of autism, Trends Cogn. Sci. 6 (2002) 248-254.

72. N. L. Johnson, S. Kotz and A. W. Kemp, Univariate Discrete Distributions (Wiley-Interscience, Hoboken, NJ, 1993).

73. M. V. Lombardo, E. Ashwin, B. Auyeung, B. Chakrabarti, K. Taylor, G. Hackett et al., Fetal testosterone influences sexually dimorphic grey matter in the human brain, J. Neurosci. 32 (2012) 674680.

74. S. Bejerot, J. M. Eriksson, S. Bonde, K. Carlstrom, M. B. Humble and E. Eriksson The extreme male brain revisited: Gender coherence in adults with autism spectrum disorder, Br. J. Psychiatry 201 (2012) 116-123.

75. F. J. Martinez-Murcia, M. C. Lai, J. M. Górriz, J. Ramírez, A. M. Young, S. C. Deoni and C. Ecker, M. V. Lombardo, M. R. C. AIMS Consortium, S. Baron-Cohen, D. G. Murphy, E. T. Bullmore and J. Suckling, On the brain structure heterogeneity of autism: Parsing out acquisition site effects with significance-weighted principal component analysis, Hum. Brain Mapp. 38(3) (2017) 1208-1223, doi: $10.1002 / \mathrm{hbm} .23449$.

76. F. Segovia, R. Holt, M. Spencer, J. M. Górriz, J. Ramírez, C. G. Puntonet, C. Phillips, L. Chura, S. Baron-Cohen and J. Suckling, Identifying endophenotypes of autism: A multivariate approach, Front. Comput. Neurosci. 8 (2014) 60, doi: 10.3389/fncom.2014.00060.

77. A. S. Heinsfelda, A. R. Francob, R. C. Craddockf, A. Buchweitz and F. Meneguzzia, Identification of autism spectrum disorder using deep learning and the ABIDE dataset, NeuroImage, Clin. 17 (2018) 16-23.
78. G. Mirzaei, A. Adeli and H. Adeli, Imaging and machine learning techniques for diagnosis of alzheimer disease, Rev. Neurosci. 27(8) (2016) 857870 .

79. M. Ahmadlou, H. Adeli and A. Adeli, Fuzzy synchronization likelihood-wavelet methodology for diagnosis of autism spectrum disorder, J. Neurosci. Methods 211(2) (2012) 203-209.

80. S. Bhat, U. R. Acharya and H. Adeli, G. M. Muralidhar Bairy and A. Adeli, Automated diagnosis of autism: In search of a mathematical marker, Rev. Neurosci. 25(6) (2014) 851-861.

81. S. Bhat, U. R. Acharya, H. Adeli, G. M. Muralidhar Bairy and A. Adeli, Autism: Cause factors, early diagnosis and therapies, Rev. Neurosci. 25(6) (2014) 841-850.

82. F. C. Morabito, M. Campolo, N. Mammone, M. Versaci, S. Franceschetti, F. Tagliavini, V. Sofia, D. Fatuzzo, A. Gambardella, A. Labate, L. Mumoli, G. G. Tripodi, S. Gasparini, V. Cianci, C. Sueri, E. Ferlazzo and U. Aguglia, Deep learning representation from electroencephalography of early-stage Creutzfeld-Jakob disease and features for differentiation from rapidly progressive dementia, Int. J. Neural Syst. 27(2) (2017) 1650039.

83. A. Antoniades, L. Spyrou, D. Martin-Lopez, A. Valentin, G. Alarcon, S. Sanei and C. C. Took, Deep neural architectures for mapping scalp to intracranial EEG, Int. J. Neural Syst. 28(8) (2018) 1850009.

84. S. Wang, Y. Hu and Y. Shen, Classification of diffusion tensor metrics for the diagnosis of a myelopathic cord using machine learning, Int. J. Neural Syst. 28(2) (2018) 1750036.

85. X. Li, Y. Bai, Y. Peng, S. Du and S. Ying, Nonlinear semi-supervised metric learning via multiple kernel and local topology, Int. J. Neural Syst. 28(2) (2018) 1750040 . 